\title{
Effect of Probiotics on Central Nervous System Functions in Animals and Humans: A Systematic Review
}

\author{
Huiying Wang, ${ }^{1,2,3}$ In-Seon Lee, ${ }^{1,2,3}$ Christoph Braun, ${ }^{2,4}$ and Paul Enck ${ }^{1 *}$ \\ ${ }^{I}$ Department of Psychosomatic Medicine and Psychotherapy, University of Tübingen, Germany; ${ }^{2}$ MEG Center, University Hospital Tübingen, \\ Germany; ${ }^{3}$ Graduate Training Center of Neuroscience, IMPRS for Cognitive and Systems Neuroscience, Tübingen, Germany; and ${ }^{4}$ CIMeC, Center \\ for Mind/Brain Sciences, University of Trento, Italy
}

To systematically review the effects of probiotics on central nervous system function in animals and humans, to summarize effective interventions (species of probiotic, dose, duration), and to analyze the possibility of translating preclinical studies. Literature searches were conducted in Pubmed, Medline, Embase, and the Cochrane Library. Only randomized controlled trials were included. In total, 38 studies were included: 25 in animals and 15 in humans (2 studies were conducted in both). Most studies used Bifidobacterium (eg, B. longum, B. breve, and B. infantis) and Lactobacillus (eg, L. helveticus, and L. rhamnosus), with doses between $10^{9}$ and $10^{10}$ colonyforming units for 2 weeks in animals and 4 weeks in humans. These probiotics showed efficacy in improving psychiatric disorderrelated behaviors including anxiety, depression, autism spectrum disorder (ASD), obsessive-compulsive disorder, and memory abilities, including spatial and non-spatial memory. Because many of the basic science studies showed some efficacy of probiotics on central nervous system function, this background may guide and promote further preclinical and clinical studies. Translating animal studies to human studies has obvious limitations but also suggests possibilities. Here, we provide several suggestions for the translation of animal studies. More experimental designs with both behavioral and neuroimaging measures in healthy volunteers and patients are needed in the future.

(J Neurogastroenterol Motil 2016;22:589-605)

Key Words

Animals; Anxiety; Depression; Humans; Probiotics

\section{Introduction}

The microbiota, the ecological community of commensal, symbiotic, and pathogenic microorganisms literally sharing our body space, includes more than 10 times the number of host cells to human cells. ${ }^{1}$ The majority of the microbiome lives in the gastrointes- tinal (GI) tract and is composed of 10100 trillion microorganisms, containing 100 times as many genes as our genome. ${ }^{2}$ Symbiosis of the gut microbiota (GM) can maintain a normal physiology in the host, while dysbiosis of the GM can shift the balance and may induce diseases.

Recent studies have found a role for the GM in the gut-brain axis, which can alter minds and behaviors through the central ner-

Received: January 27, 2016 Revised: April 25, 2016 Accepted: May 18, 2016

() This is an Open Access article distributed under the terms of the Creative Commons Attribution Non-Commercial License (http://creativecommons. org/licenses/by-nc/4.0) which permits unrestricted non-commercial use, distribution, and reproduction in any medium, provided the original work is properly cited.

*Correspondence: Paul Enck, PhD

Department of Psychosomatic Medicine and Psychotherapy, University Hospital Tübingen, Osianderstr. 5, 72076 Tübingen,

Germany

Tel: +49-7071-2989118, Fax: +49-7071-294382, E-mail: paul.enck@uni-tuebingen.de 
vous system (CNS). ${ }^{3-8}$ Maintaining GM symbiosis is important for retaining healthy CNS functions. Sudo's study was the first linking the GM and CNS, showing an increased hypothalamic-pituitaryadrenal (HPA) stress response and decreased brain-derived neurotrophic factor (BDNF) levels in the hippocampus of germfree (GF) mice. ${ }^{9}$ Recently, researchers have found a relationship between the GM and CNS-related disorders in humans, such as Parkinson's disease and autism: overall diversity and individual genus abundances were associated with their symptoms. ${ }^{10-12}$

Probiotics are defined as "live micro-organisms which, when administered in adequate amounts, confer a health benefit on the host". ${ }^{13,14}$ Some types of probiotics have been used to treat gastrointestinal disorders, such as irritable bowel syndrome (IBS). ${ }^{15-18}$ Also, probiotics have been studied in relation to altering visceral pain responses. ${ }^{19}$ Recently they have been reported to have an influence in the CNS by altering the GM composition. ${ }^{3,8}$ Studies using probiotics to change CNS functions have increased over the last 10 years. The CNS functions mostly reported being altered by probiotics are psychiatric disorders and memory abilities. Studies in animals and humans have found, and reviews have summarized, potential mechanisms underlining these probiotic effects (Fig. 1). First, probiotics may directly alter CNS biochemistry, such as by affecting levels of BDNF, $\gamma$-aminobutyric acid (GABA), serotonin (5 hydroxytryptamine; $5 \mathrm{HT}$ ), and dopamine (DA), thus influencing mind and behavior. ${ }^{20-24}$ Both the vagus and the enteric nerves are involved in this gut-brain interaction and can be affected by certain probiotics. $^{22,23}$ The HPA stress response, which regulates mood and emotion, has frequently been shown to be attenuated by probiotics, decreasing corticosteroid (CORT) levels. ${ }^{25}$ The immune system can be influenced by probiotics, limiting pro-inflammatory cytokine production and inflammation, which, in turn, can affect the endocrine and nervous systems. ${ }^{26,27}$ Probiotics manipulate GM by increasing microbiota diversity and beneficial bacteria compositions. ${ }^{28-30}$ Improved GM changes metabolites, such as short-chain fatty acids and tryptophan, which can indirectly improve CNS function. $^{26,31}$

While there are reviews describing effects of probiotics on CNS function, there has been no previous systematic review that analyzes all the current animal and human studies in the field and describes the most effective probiotic interventions. Furthermore, animal studies in this area outnumber human studies, because behavioral experiments in animals are better established and standardized, while clinical studies in humans on this topic started to increase a few years ago and need translation from preclinical studies. How-

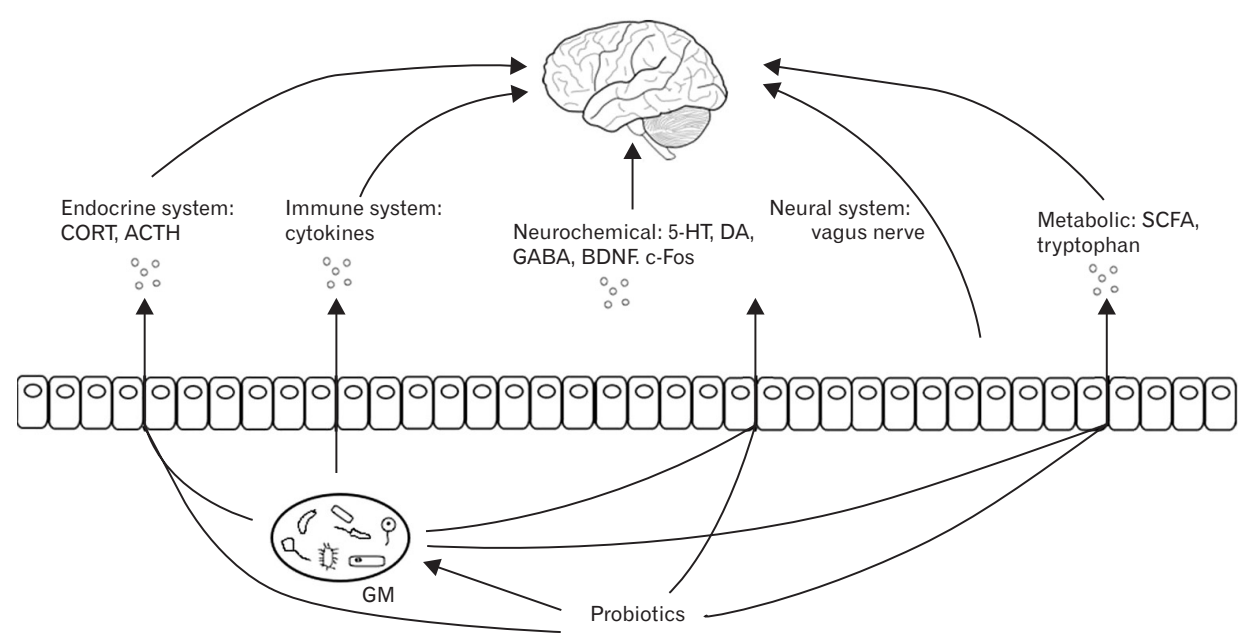

Figure 1. Mechanisms of probiotic effects on the central nervous system. Probiotics influence central nervous system (CNS) function through direct and indirect mechanisms. Probiotics affect the hypothalamic-pituitary-adrenal (HPA) axis, by altering corticosteroid (CORT) and/or adrenocorticotropic hormone (ACTH) levels. The immune system is influenced by limited pro-inflammatory cytokine production and inflammation, and this, in turn, has effects on the CNS. Probiotics can also directly alter CNS biochemistry, such as by affecting brain-derived neurotrophic factor (BDNF), c-Fos, $\gamma$-aminobutyric acid (GABA), 5 hydroxytryptamine (5-HT), and dopamine (DA) levels, thus influencing mind and behavior. The vagus and enteric nerves are also involved in this gut-brain interaction and are affected by certain probiotics. Probiotics manipulate the gut microbiota (GM) by increasing microbiota diversity and beneficial bacteria composition. An "improved" GM changes metabolites, such as shortchain fatty acids (SCFAs) and tryptophan, and so improves CNS function indirectly. The GM also interacts with the endocrine, immune, and neural systems. 
ever, from preclinical animal models to clinical trials in humans, there is no direct translation. To bridge the gap between preclinical and clinical studies, a systematic review is needed to summarize effective probiotic interventions on CNS function. We first sought to describe CNS functions that can be influenced by probiotics. Second we provide information about probiotic interventions including strain, dosage, and duration. Furthermore, we analyze and discuss the potential translation of animal models to human studies.

\section{Methods}

\section{Search Strategy}

This systematic review was conducted according to guidelines of the "Cochrane Handbook for Systematic Reviews of Interventions", following the Preferred Items for Systematic Reviews and Meta-analysis guidelines. ${ }^{32,33}$ Relevant studies were found by searches in the Pubmed, Medline, Embase, and Cochrane Library databases. Articles from 1950 to April 2016 were initially searched using the search terms "(probiotic OR gut microbiota) AND (behavior OR central nervous system)". Additional citations were sought using references in articles retrieved from searches. We only included articles written in English. The first search was undertaken by analyzing text words contained in the title and abstract, and of the key words describing the articles. The second search was conducted according to the citations from all identified reports and relevant review articles.

\section{Study Selection}

We included all animal and human studies using different strains of probiotics. In human studies reports of both healthy volunteers and patients were considered.

The abstracts of the retrieved papers were screened for matching the following criteria: (1) the study included a probiotic intervention and (2) the study tested CNS function. After exclusion of non-relevant studies, the remaining articles were screened for the following criteria: (1) the study was described as a randomized controlled study (RCT), (2) the study was described as double-blind if studied in human participants, (3) the study involved use of probiotics in single- and/or multi-strains and those that only used prebiotics or antibiotics were not included, and (4) the study included measures of behavioral experiments, neuropsychological measures (eg, electroencephalography, magnetoencephalography, and functional magnetic resonance imaging). Those that only involved neurophysiological measures were not included in the qualitative analy- sis but only in the Discussion (eg, measuring neurochemical level, HPA axis activity) because different studies tested quite divergent aspects of lower-level CNS activity.

\section{Data Collection and Analysis}

A data extraction and assessment form from the Cochrane Collaboration was used to further exclude inappropriate studies and to extract data we needed to analyze. ${ }^{33}$ Double extraction of data was conducted: important data included the source of participants (animal species/strains, patient types), intervention groups (probiotic, placebo, or any other intervention), sample size, duration of interventions, and outcomes (behavioral changes as the primary outcome and lower-level changes [eg, biochemical changes] as secondary outcomes).

We assessed the quality of each study included using the Quality Assessment Tool for Quantitative Studies and the Quality Assessment Tool for Quantitative Studies Dictionary developed by The Effective Public Health Practice Project (EPHPP) (National Collaborating Centre for Methods and Tools [2008]). ${ }^{34}$

The inclusion of both animals and human studies resulted in great heterogeneity of participants, interventions, and CNS functions measured. The outcomes of each study were described. A qualitative synthesis of selected studies was made with the aim of coming to conclusions about which probiotic interventions, at which dose, and for how long, were more/most effective with regard to certain CNS functions. Because of the heterogeneity of designs a meta-analysis was deemed to be inappropriate.

\section{Results}

In total, 46 potentially relevant citations were obtained through the primary search strategy, which included animal and human studies, after excluding reviews $(\mathrm{n}=98)$ (systematic reviews, narrative reviews, respective reviews, and systematic reviews and meta-analyses), other articles including comments, hypotheses, viewpoints, opinions, and editorials $(\mathrm{n}=13)$, studies without the use of a probiotic ( $\mathrm{n}=33$ ), and studies not focusing on CNS function $(\mathrm{n}=16)$ (Fig. 2). Many articles concerned the potential, and demonstrated mechanisms of the effects, of GM and/or probiotics on CNS function. However, we only included studies that clearly described probiotics as interventions, and excluded studies only measuring GM composition when investigating CNS function. After the full screen, 8 more studies were excluded: four studies lacked a control arm or blinding, and 4 studies did not measure CNS functions at the behavioral level. In total, 38 studies remained for 


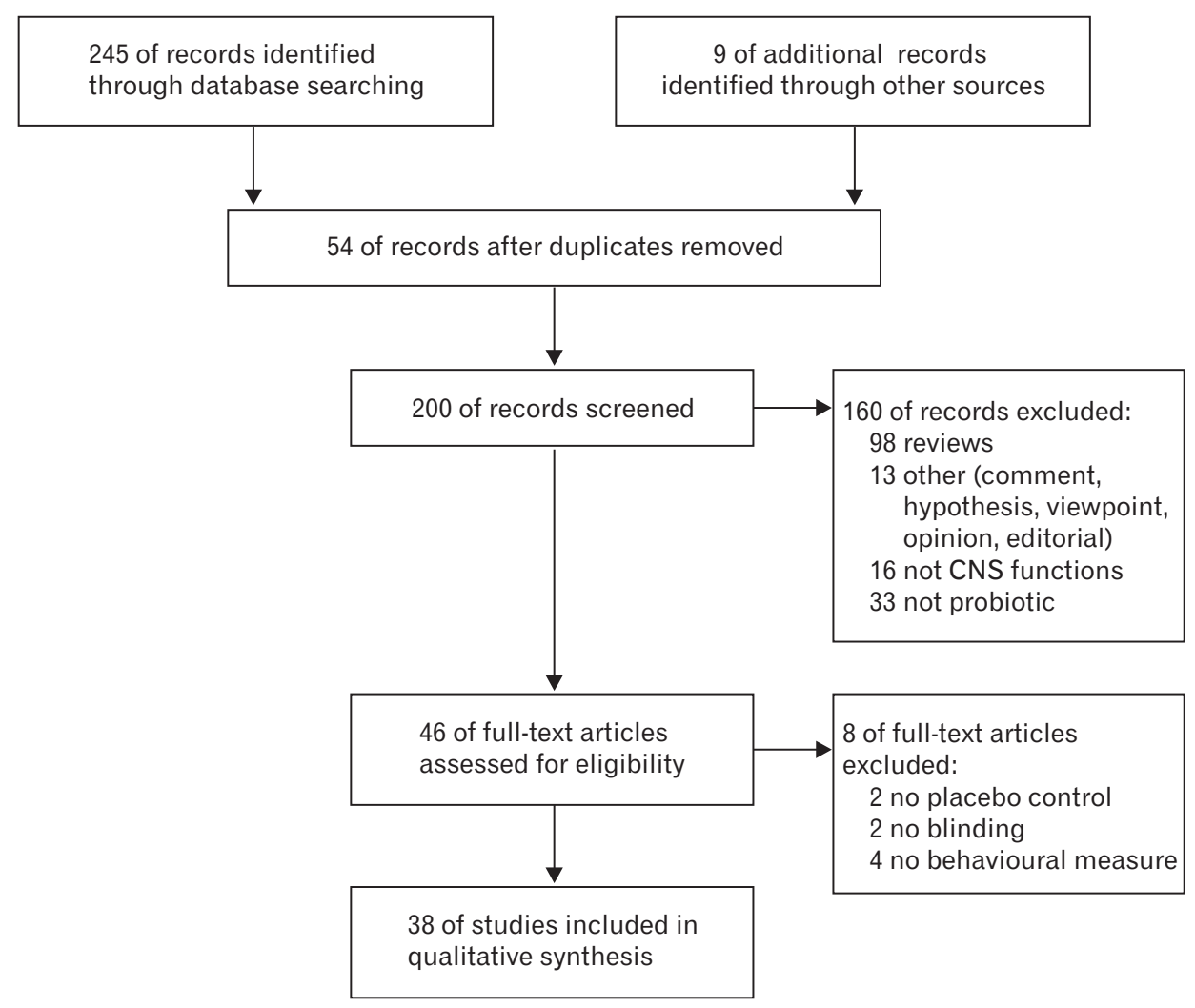

Figure 2. Preferred reporting items for systematic reviews and meta-analyses (PRISMA) scheme of retrieved literature. CNS, central nervous system. the qualitative analysis. Of them, 15 were in humans and 25 were in animals (2 studies were conducted in humans and animals).

\section{Animal Studies}

Of the 25 studies, 19 provided explicit information in the Materials and Methods section on the numbers of animals allocated to each treatment group, and they accounted for all the animals in their results, while 6 studies provided specifics regarding treated animals only in the pertinent areas of the Results section. Results from the quality assessment tool for quantitative studies showed all studies were strong in global ratings for selection bias, study design, confounders, blinding, data collection method, and withdrawals and dropouts (no weak rating): 15 studies described assigning animals randomly to treatment groups or control, although these animal studies were supposed to be RCTs. Only four studies might have confounders because they used both male and female animals while the other studies only used single gender animals, excluding the effect of gender on the results. Only 13 studies reported that experimenters were blinded toward the interventions or exposure status of the participants (we included the other 12 studies that did not describe blinding, because animals were not aware of the research question or intervention, and we ignored potential effects of the experimenter). Data collection tools shown in all studies were considered valid and reliable; no study reported withdrawals or drop outs.

Although all of studies examined were on rodents (mice or rats), the selection of animals was heterogeneous in some respects: strains of animals and health conditions of the animals. Studies were also heterogeneous in the CNS functions they were looking at, the experimental models they used, the probiotics they used, and the dose and duration of the probiotic interventions. Due to the heterogeneity of the included studies, we only described the results based on the interventions and measurements of the CNS functions (Table 1).

Most of the studies (18/25) investigated the effects of a single strain of probiotics. In 18 studies using single-strain probiotics, seven used Bifidobacterium, eleven used Lactobacillus, and one used Clostridium (one used both Bifidobacterium and Lactobacillus). Almost all the studies found significant effects on measured CNS functions, except for one testing effect of Bifidobacterium infantis on depression-like behavior. The concentration of the effective probiotic interventions ranged from $10^{7}$ to $10^{11}$ colony-forming units (CFU), with the most using $10^{9}(14 / 25)$ or $10^{10}(6 / 25) \mathrm{CFU}$ per animal per day. The duration of the probiotic treatments ranged 
Table 1. Studies of Effects of Probiotics on Central Nervous System Functions in Animals

\begin{tabular}{|c|c|c|c|c|c|}
\hline Study & Animal & CNS function & $\begin{array}{c}\text { Probiotic } \\
\text { (species, dosage } \\
[\mathrm{CFU} / \text { day }], \text { duration })\end{array}$ & $\begin{array}{c}\text { Outcome } \\
\text { (behavioral level) }\end{array}$ & Secondary outcome \\
\hline $\begin{array}{l}\text { Liu et al, } \\
2016\end{array}$ & $\begin{array}{l}\text { ELS mice } \\
\text { Naïve mice }\end{array}$ & $\begin{array}{l}\text { Locomotor activity } \\
\text { (open-field test) } \\
\text { Anxiety (open-field test, } \\
\text { elevated plus maze) } \\
\text { Depression (sucrose- } \\
\text { preference test, } \\
\text { forced-swimming test) }\end{array}$ & $\begin{array}{l}\text { L. plantarum PS128 } \\
10^{9} \\
28 \text { days }\end{array}$ & $\begin{array}{l}\text { Locomotor activity } \uparrow \\
\text { Anxiety (only in naïve } \\
\text { mice) } \downarrow \\
\text { Depression (only in ELS } \\
\text { mice) } \downarrow\end{array}$ & $\begin{array}{l}\text { Corticosteroids (CORT) } \downarrow \\
\text { Cytokine: inflammatory cytokine } \\
\text { TNF- } \alpha, \text { IL- } 6 \downarrow \text {, anti-inflammatory } \\
\text { cytokine IL- } 10 \uparrow \\
\text { Brain monoamines and metabo- } \\
\text { lites: } 5 \text {-HT } \uparrow, 5 \text {-HIAA } \downarrow \text {, DA } \uparrow, \\
\text { DOPAC } \downarrow, \text { HVA } \downarrow\end{array}$ \\
\hline $\begin{array}{l}\text { Liu et al, } \\
2016\end{array}$ & GF mice & $\begin{array}{l}\text { Locomotor activity } \\
\text { (open-field test) } \\
\text { Anxiety (elevated-plus } \\
\text { maze) } \\
\text { Depression } \\
\text { (forced-swimming test) }\end{array}$ & $\begin{array}{l}\text { L. plantarum PS128 } \\
10^{9} \\
16 \text { days }\end{array}$ & $\begin{array}{l}\text { Locomotor activity } \uparrow \\
\text { Anxiety } \downarrow \\
\text { Depression (-) }\end{array}$ & $\begin{array}{l}\text { CORT: NA } \\
\text { Brain monoamines and } \\
\text { metabolites: DA } \uparrow, \mathrm{HVA} \uparrow, 5 \text {-HT } \uparrow \\
\text { and 5-HIAA } \uparrow\end{array}$ \\
\hline $\begin{array}{l}\text { Liang et al, } \\
2015\end{array}$ & SPF CRS rats & $\begin{array}{l}\text { Stress (chronic-restraint } \\
\text { stress) } \\
\text { Depression (sucrose- } \\
\text { preference test) } \\
\text { Anxiety (elevated-plus } \\
\text { maze, open-field test) } \\
\text { Non-spatial memory } \\
\text { (object-recognition test, } \\
\text { object-placement test) }\end{array}$ & $\begin{array}{l}\text { L. helveticus NS8 } \\
10^{9} \\
\text { Initial } 21 \text { days }\end{array}$ & $\begin{array}{l}\text { Anxiety } \downarrow \\
\text { Depression } \downarrow \\
\text { Non-spatial memory } \uparrow\end{array}$ & $\begin{array}{l}\text { CORT and ACTH } \downarrow \\
\text { Cytokines: IL-10 } \uparrow \\
\text { Brain monoamines: } 5 \text {-HT } \uparrow \text { and } \\
\text { NE } \uparrow \\
\text { BDNF expression } \uparrow\end{array}$ \\
\hline $\begin{array}{l}\text { Wang et al, }{ }^{30} \\
2015\end{array}$ & $\begin{array}{l}\text { Ampicillin-treated } \\
\text { rats }\end{array}$ & $\begin{array}{l}\text { Anxiety (elevate-plus } \\
\text { maze) } \\
\text { Spatial memory } \\
\text { (Morris water maze) }\end{array}$ & $\begin{array}{l}\text { L. fermentum N93 } \\
10^{9} \\
\text { Initial } 30 \text { days }\end{array}$ & $\begin{array}{l}\text { Anxiety } \downarrow \\
\text { Spatial memory } \uparrow\end{array}$ & $\begin{array}{l}\text { CORT and ACTH } \downarrow \\
\text { Brain monoamines: MR } \uparrow, \\
\text { NMDA } \uparrow, \text { GR: NA } \\
\text { Brain BDNF: NA } \\
\text { Colon inflammation: } \\
\text { myeloperoxidase activity } \downarrow \\
\text { Fecal microbiota: Bacteroides } \uparrow \text {, } \\
\text { C. coccoides } \downarrow, \text { Firmicutes } \downarrow \text {, } \\
\text { Lactobacillus } \uparrow\end{array}$ \\
\hline $\begin{array}{l}\text { Smith et al, }{ }^{29} \\
2014\end{array}$ & $\mathrm{RagI}^{--}$mice & $\begin{array}{l}\text { Stress response } \\
\text { (water-avoidance stress) } \\
\text { Anxiety (light/dark } \\
\text { box test) } \\
\text { Non-spatial memory } \\
\text { (novel- object test) }\end{array}$ & $\begin{array}{l}\text { L. rhamnosus } \mathrm{R} 0011+ \\
\text { L. helveticus } \mathrm{R} 0052 \\
6 \times 10^{9} \\
28 \text { days }\end{array}$ & $\begin{array}{l}\text { Anxiety } \downarrow \\
\text { Non-spatial memory } \uparrow\end{array}$ & $\begin{array}{l}\text { CORT: NA } \\
\text { Brain c-Fos expression } \uparrow \\
\text { Intestinal permeability } \downarrow \\
\text { Fecal microbiota: Bacteroides } \uparrow, \\
\text { Enterobacteriaceae } \uparrow, \text { Firmicutes } \uparrow\end{array}$ \\
\hline $\begin{array}{l}\text { Luo et al, } \\
2014\end{array}$ & $\begin{array}{l}\text { Hyperammone- } \\
\text { mia rats }\end{array}$ & $\begin{array}{l}\text { Anxiety (elevate-plus } \\
\text { maze) } \\
\text { Spatial memory } \\
\text { (Morris water maze) }\end{array}$ & $\begin{array}{l}\text { L. helveticus NS8 } \\
10^{9} \\
14 \text { days }\end{array}$ & $\begin{array}{l}\text { Anxiety } \downarrow \\
\text { Spatial memory } \uparrow\end{array}$ & $\begin{array}{l}\text { Neuroinflammation: PGE2 } \downarrow \text {, } \\
\text { IL-1 } \beta \downarrow \\
\text { Brain monoamines: } 5 \text {-HT } \downarrow \\
\text { Plasma kynurenine pathway: } \\
\text { KYN/TRP } \uparrow, \text { KA/KYN } \downarrow\end{array}$ \\
\hline $\begin{array}{l}\text { Savignac et } \\
\mathrm{al}^{36} 2014\end{array}$ & Mice & $\begin{array}{l}\text { Anxiety (defensive marble } \\
\text { burying, elevated-plus } \\
\text { maze, open field) } \\
\text { depression (tail-suspen- } \\
\text { sion test, forced-swim } \\
\text { test) }\end{array}$ & $\begin{array}{l}\text { B. longum 1714/ } \\
\text { B. breve } 1205 \\
10^{9} \\
\text { Initial } 21 \text { days }\end{array}$ & $\begin{array}{l}\text { B. longum: } \\
\text { Anxiety } \downarrow \\
\text { Depression } \downarrow \\
\text { B. breve: } \\
\text { Anxiety } \downarrow\end{array}$ & CORT: NA \\
\hline $\begin{array}{l}\text { Ohland et } \\
\mathrm{al}^{37} 2013\end{array}$ & $\begin{array}{l}\text { Il-10 deficient } \\
\text { mice }\end{array}$ & $\begin{array}{l}\text { Anxiety (elevated } \\
\text { Barnes Maze) } \\
\text { Spatial memory } \\
\text { (elevated Barnes maze) }\end{array}$ & $\begin{array}{l}\text { L. helveticus R0052 } \\
10^{9} \\
21 \text { days }\end{array}$ & $\begin{array}{l}\text { Anxiety } \downarrow \\
\text { Spatial memory } \uparrow\end{array}$ & $\begin{array}{l}\text { Colon inflammation } \downarrow \\
\text { Cytokines: NA } \\
\text { CORT } \downarrow \\
\text { SCFA metabolites: NA }\end{array}$ \\
\hline
\end{tabular}


Table 1. Continued

\begin{tabular}{|c|c|c|c|c|c|}
\hline Study & Animal & CNS function & $\begin{array}{c}\text { Probiotic } \\
\text { (species, dosage } \\
[\mathrm{CFU} / \mathrm{day}], \text { duration })\end{array}$ & $\begin{array}{c}\text { Outcome } \\
\text { (behavioral level) }\end{array}$ & Secondary outcome \\
\hline $\begin{array}{l}\text { Messaoudi } \\
\text { et al, }{ }^{39} 2011\end{array}$ & Rats & $\begin{array}{l}\text { Anxiety (conditioned } \\
\text { defensive burying) }\end{array}$ & $\begin{array}{l}\text { B. longum R0175 + } \\
\text { L. helveticus R0052 } \\
10^{9} \\
14 \text { days }\end{array}$ & Anxiety $\downarrow$ & NA \\
\hline $\begin{array}{l}\text { Bravo et al, } \\
2011\end{array}$ & Mice & $\begin{array}{l}\text { Anxiety (open arms, } \\
\text { elevated-plus maze, } \\
\text { fear conditioning) } \\
\text { Depression } \\
\text { (forced-swim test) }\end{array}$ & $\begin{array}{l}\text { L. rhamnosus JB-1 } \\
10^{9} \\
28 \text { days }\end{array}$ & $\begin{array}{l}\text { Anxiety } \downarrow \\
\text { Depression } \downarrow\end{array}$ & $\begin{array}{l}\text { CORT } \downarrow \\
\text { GABA receptor expression influence } \\
\text { depending on brain areas } \\
\text { Probiotic effect via vagus nerve }\end{array}$ \\
\hline $\begin{array}{l}\text { Bercik et } \\
\mathrm{al}^{22} 2011\end{array}$ & $\begin{array}{l}\text { Chronic colitis } \\
\text { mice }\end{array}$ & Anxiety (step-down test) & $\begin{array}{l}\text { B. longum NCC } 3001 \\
10^{10} \\
14 \text { days }\end{array}$ & Anxiety $\downarrow$ & $\begin{array}{l}\text { Colon inflammation: NA } \\
\text { Brain BDNF expression: NA } \\
\text { Enteric neurons excitability } \downarrow \\
\text { Probiotic effect via vagus nerve }\end{array}$ \\
\hline $\begin{array}{l}\text { Bercik et } \\
\mathrm{al},{ }^{35} 2010\end{array}$ & $\begin{array}{l}\text { T-muris infected } \\
\text { mice }\end{array}$ & $\begin{array}{l}\text { Anxiety (light/dark } \\
\text { behavior, step-down test) }\end{array}$ & $\begin{array}{l}\text { B. longum NCC } 3001 / \\
\text { L. rhamnosus NCC4007 } \\
10^{10} \\
10 \text { days }\end{array}$ & Anxiety $\downarrow$ (B. longum only) & $\begin{array}{l}\text { Colon inflammation } \downarrow \\
\text { Plasma cytokines: NA } \\
\text { BDNF expression } \uparrow \\
\text { (only by B. longum) } \\
\text { Tryptophan and kynurenine: NA } \\
\text { No effect of vagotomy }\end{array}$ \\
\hline $\begin{array}{l}\text { Singh et al, } \\
2012\end{array}$ & Rats & $\begin{array}{l}\text { CFS and depression } \\
\text { induced by forced-swim } \\
\text { test (immobility period, } \\
\text { post-swim fatigue time) }\end{array}$ & $\begin{array}{l}\text { L. acidophilus as LAB } \\
\text { or LAB FB } \\
10^{7} \\
7 \text { days }\end{array}$ & $\begin{array}{l}\text { Depression } \downarrow \text { (larger effect } \\
\text { of LAB FB than LAB); }\end{array}$ & $\begin{array}{l}\text { Brain oxido-nitrosative stress } \\
\text { biomarker } \downarrow \\
\text { Cytokines: TNF- } \alpha \downarrow\end{array}$ \\
\hline $\begin{array}{l}\text { Arseneautl- } \\
\text { Bread et } \\
\text { al, }^{41} 2012\end{array}$ & MI rats & $\begin{array}{l}\text { Post-MI depression } \\
\text { (forced-swim test); } \\
\text { social behavior } \\
\text { (social interaction test); } \\
\text { emotional memory } \\
\text { (passive avoidance } \\
\text { step-down test) }\end{array}$ & $\begin{array}{l}\text { B. longum R } 0175+ \\
\text { L. helveticus R0052 } \\
10^{9} \\
14 \text { days }\end{array}$ & $\begin{array}{l}\text { Depression } \downarrow \\
\text { Social interaction } \uparrow \\
\text { Non-spatial memory } \uparrow\end{array}$ & $\begin{array}{l}\text { Cytokines: pro-inflammatory } \\
\text { cytokine IL-1 } \downarrow \\
\text { Intestinal barrier permeability } \downarrow\end{array}$ \\
\hline $\begin{array}{l}\text { Desbonnet } \\
{\text { et } \mathrm{al}^{27}}^{27} 2010\end{array}$ & MS rats & $\begin{array}{l}\text { Depression } \\
\text { (forced-swim test) }\end{array}$ & $\begin{array}{l}\text { B. infantis } 35624 \\
10^{10} \\
\text { Initial } 40 \text { days }\end{array}$ & Depression $\downarrow$ & $\begin{array}{l}\text { CORT: NA } \\
\text { Cytokines: IL-10 } \downarrow \\
\text { Tryptophan: NA } \\
\text { Brain monoamines (-) } \\
\text { Noradrenaline } \uparrow\end{array}$ \\
\hline $\begin{array}{l}\text { Desbonnet } \\
{\text { et } \mathrm{al}^{26}}^{26} 2008\end{array}$ & Rats & $\begin{array}{l}\text { Depression } \\
\text { (forced-swim test) }\end{array}$ & $\begin{array}{l}\text { B. infantis } 35624 \\
10^{10} \\
14 \text { days }\end{array}$ & No behavioral change & $\begin{array}{l}\text { Cytokines: pro-inflammatory cytokines } \\
\text { IL-6, IFN- } \gamma \downarrow \text {; anti-inflammatory } \\
\text { cytokines IL-10 } \downarrow \\
\text { Tryptophan } \uparrow \\
\text { Brain monoamines and metabolites - } \\
\text { 5-HIAA } \downarrow, \text { DOPAC } \downarrow \\
\text { Neuroendocrine: NA, CORT: NA, } \\
\text { AVP: NA, CRF: NA }\end{array}$ \\
\hline $\begin{array}{l}\text { Liu etal, } \\
2015\end{array}$ & $\begin{array}{l}\mathrm{VaD} \text { (vascular } \\
\text { dementia) mice }\end{array}$ & $\begin{array}{l}\text { Locomotor activity } \\
\text { (open-field test) } \\
\text { Spatial memory } \\
\text { (Morris water maze) }\end{array}$ & $\begin{array}{l}\text { C. butyricum } \\
\text { WZMC1016 } \\
(\text { CGMCC } 9831) \\
10^{6} / 10^{7} / 10^{8} \\
42 \text { days }\end{array}$ & $\begin{array}{l}\text { Locomotor activity } \uparrow \\
\text { Spatial memory } \uparrow\end{array}$ & $\begin{array}{l}\text { Morphological change of } \\
\text { hippocampus } \downarrow \\
\text { BDNF expression } \uparrow \\
\text { Butyrate in feces and brain } \uparrow \\
\text { Fecal bacteria diversity } \uparrow\end{array}$ \\
\hline $\begin{array}{l}\text { Jeong et al, } \\
2015\end{array}$ & Aged rats & $\begin{array}{l}\text { Spatial memory (Y-maze, } \\
\text { Morris water maze) }\end{array}$ & $\begin{array}{l}\text { L. plantarum KY1032 + } \\
\text { L. curvatus HY7601 } \\
10^{10} \\
48 \text { days }\end{array}$ & Spatial memory $\uparrow$ & $\begin{array}{l}\text { Cytokines: pro-inflammatory } \\
\text { cytokines NF- } \mathrm{B}, \downarrow \\
\text { BDNF } \uparrow \\
\text { Lipidemia } \downarrow\end{array}$ \\
\hline
\end{tabular}


Table 1. Continued

\begin{tabular}{|c|c|c|c|c|c|}
\hline Study & Animal & CNS function & $\begin{array}{c}\text { Probiotic } \\
\text { (species, dosage } \\
[\mathrm{CFU} / \text { day }] \text {, duration })\end{array}$ & $\begin{array}{c}\text { Outcome } \\
\text { (behavioral level) }\end{array}$ & Secondary outcome \\
\hline $\begin{array}{l}\text { Savignac et } \\
\mathrm{al}^{43} 2015\end{array}$ & Mice & $\begin{array}{l}\text { Non-spatial memory } \\
\text { (object recognition, } \\
\text { fear conditioning) } \\
\text { Spatial memory } \\
\text { (Barnes Maze) }\end{array}$ & $\begin{array}{l}\text { B. longum } 1714 \text { / } \\
\text { B. breve } 1205 \\
10^{9} \\
\text { initial } 21 \text { days }\end{array}$ & $\begin{array}{l}\text { B. longum: } \\
\text { Non-spatial memory } \uparrow \\
\text { Spatial memory } \uparrow \\
\text { B. breve: } \\
\text { Non-spatial memory } \uparrow\end{array}$ & $\begin{array}{l}\text { Visceral sensitivity -colon distension: } \\
\text { NA } \\
\text { CORT: NA }\end{array}$ \\
\hline $\begin{array}{l}\text { Gareau et } \\
\mathrm{al}^{45} 2011\end{array}$ & $\begin{array}{l}\text { C. rodentium } \\
\text { infected mice }\end{array}$ & $\begin{array}{l}\text { Memory dysfunction } \\
\text { induced by water } \\
\text { avoidance (novel-object } \\
\text { test, T-maze); }\end{array}$ & $\begin{array}{l}\text { L. rhamnosus } \mathrm{R} 0011+ \\
\text { L. helveticus } \mathrm{R} 0052 \\
6 \times 10^{9} \\
17 \text { days }\end{array}$ & Non-spatial memory $\uparrow$ & $\begin{array}{l}\text { CORT } \downarrow \\
\text { Colon epithelial cell hyperplasia } \downarrow \\
\text { Cytokine: pro-inflammatory } \\
\text { cytokines IFN } \gamma \downarrow \\
\text { Brain BDNF and c-Fos expression } \uparrow \\
\text { Microbiota: Firmicutes } \downarrow \text {, } \\
\text { Enterobacteriaceae } \downarrow \text {, Eubacteria } \\
\text { rectale } \downarrow \text {, Lactobacillus } \uparrow\end{array}$ \\
\hline $\begin{array}{l}\text { Davari et } \\
\mathrm{al}^{46} 2013\end{array}$ & Diabetic rats & $\begin{array}{l}\text { Spatial memory } \\
\text { (Morris water maze) }\end{array}$ & $\begin{array}{l}\text { L. acidophilus } 4356+ \\
\text { B. lactis } 10140+ \\
\text { L. fermentum } \\
\text { ATCC } 9338 \\
2 \times 10^{10} \\
56 \text { days }\end{array}$ & Spatial memory $\uparrow$ & $\begin{array}{l}\text { Hippocampal long-term potentiation } \uparrow \\
\text { Serum glucose } \downarrow \text { and insulin } \uparrow \\
\text { Oxidative stress biomarkers: SOD } \uparrow \text {, } \\
\text { 8-OHdG } \downarrow\end{array}$ \\
\hline $\begin{array}{l}\text { Hsiao et } \\
\mathrm{al}^{31} 2013\end{array}$ & MIA mice & $\begin{array}{l}\text { Autism spectrum disorder: } \\
\text { Anxiety (open field, } \\
\text { marble burying); } \\
\text { Sensory gating } \\
\text { (prepulse inhibition); } \\
\text { Communicative behavior } \\
\text { (ultrasonic vocalizations); } \\
\text { Social interaction } \\
\text { (3-chamber social test) }\end{array}$ & $\begin{array}{l}\text { B. fragilis NCTC } 9343 \\
10^{10} \\
6 \text { days }\end{array}$ & $\begin{array}{l}\text { Anxiety } \downarrow \\
\text { Sensory gating } \uparrow \\
\text { Communicative behavior } \uparrow \\
\text { Social interaction (-) }\end{array}$ & $\begin{array}{l}\text { Intestinal permeability } \downarrow \\
\text { Tryptophan metabolites: } \\
\text { indolepyruvate } \downarrow \\
\text { Microbiota: Lachnospiraceae } \downarrow \text {, } \\
\text { Bacteroidales } \downarrow\end{array}$ \\
\hline $\begin{array}{l}\text { Kantak et } \\
\mathrm{al}^{48}{ }^{48} 2014\end{array}$ & Male mice & $\begin{array}{l}\text { obsessive-compulsive-dis- } \\
\text { order-like behavior (open } \\
\text { field, marble burying, } \\
\text { ultrasonic vocalizations, } \\
\text { intermale aggression) }\end{array}$ & $\begin{array}{l}\text { L. rhamnosus GG } \\
\text { (ATCC 53103) } \\
10^{9} \\
14,28 \text { days }\end{array}$ & $\begin{array}{l}\text { Locomotor behavior } \downarrow \\
\text { Marble burying } \downarrow \\
\text { Ultrasonic vocalizations (-) } \\
\text { Intermale aggression (-) }\end{array}$ & NA \\
\hline $\begin{array}{l}\text { D'Mello et } \\
{\mathrm{al},{ }^{49}}^{4} 2015\end{array}$ & Male mice & $\begin{array}{l}\text { Inflammation associated } \\
\text { sickness behavior } \\
\text { (social exploratory) }\end{array}$ & $\begin{array}{l}\text { VSL\#3 } \\
1.7 \times 10^{9} \\
10 \text { days }\end{array}$ & $\begin{array}{l}\text { Social exploratory behavior } \\
\text { in bile duct ligation treated } \\
\text { mice } \uparrow\end{array}$ & $\begin{array}{l}\text { Intestinal permeability: NA } \\
\text { Cytokine: pro-inflammatory cytokine } \\
\text { TNF- } \alpha \downarrow \\
\text { Monocyte infiltration } \downarrow \\
\text { Microglial activation } \downarrow \\
\text { Fecal Microbiota (-) }\end{array}$ \\
\hline $\begin{array}{l}\text { Takada et } \\
\mathrm{al}^{50} 2016\end{array}$ & Male rats & $\begin{array}{l}\text { Stress response to water } \\
\text { avoidance stress }\end{array}$ & $\begin{array}{l}\text { L. casei Shirota YIT } 9029 \\
3 \times 10^{9} \\
14 \text { days }\end{array}$ & $\mathrm{NA}$ & $\begin{array}{l}\text { CORT } \downarrow \\
\text { c-Fos expression in the paraventricular } \\
\text { nucleus } \downarrow \\
\text { Gastric vagal afferent activity } \uparrow \\
\text { Neuronal excitability of NTS } \uparrow\end{array}$ \\
\hline
\end{tabular}

ELS, early life stress; CORT, corticosterone; 5-HT, 5-hydroxytryptamine; 5-HIAA, 5-Hydroxyindoleacetic acid; DA, dopamine; DOPAC, 3,4-dihydroxyphenylacetic acid; HVA, homovanillic acid; GF, germ free; NA, not applicable; SPF, specific pathogen free; CRS, chronic restraint stress; ACTH, adrenocorticotropic hormone; NE, norepinephrine; BDNF, brain-derived neurotrophic factor; MR, mineralocorticoid; NMDA, N-methyl-D-aspertate; GR, glucocorticoid; RagI-/-, RagI knockout; PGE2, prostaglandin E2; KYN, L-kynurenine; TRP, tryptophan; KA, kynurenic acid; SCFA, short-chain fatty acid; GABA, gamma-Aminobutyric acid; CFS, chronic fatigue syndrome; LAB, Lactobacillus acidophilus; FB, floating bead; MI, myocardial infarction; MS, maternal separation; AVP, arginine vasopressin; $\mathrm{CRF}$, corticotrophin-releasing factor; $\mathrm{VaD}$, vascular dementia; NF- $\mathrm{KB}$, nuclear factor-kappa B; SOD, superoxide dismutase; 8-OHdG, 8-hydroxy2'deoxyguanosine; MIA, maternal immune activation; VSL\#3, a high-concentration probiotic preparation of 8 live freeze-dried bacterial (Lactobacillus casei, Lactobacillus plantarum, Lactobacillus acidophilus, Lactobacillus bulgaricus, Bifidobacterium longum, Bifidobacterium breve, Bifidobacterium infantis, and Streptococcus thermophiles); NTS, nucleus tractus solitary; B. breve, Bifidobacterium breve; $B$. fragilis, Bifidobacterium fragilis; $B$. infantis, Bifidobacterium infantis; B. lactis, Bifidobacterium lactis; B. longum, Bifidobacterium longum; C. butyricum, Clostridium butyricum; C. coccoides, Clostridium coccoides; C. rodentium, Citrobacter rodentium; L. acidophilus, Lactobacillus acidophilus; L. casei, Lactobacillus casei; $L$. curvatus, Lactobacillus curvatus; $L$. fermentum, Lactobacillus fermentum; L. helveticus, Lactobacillus helveticus; L. plantarum, Lactobacillus plantarum; L. rhamnosus, Lactobacillus rhamnosus. 
from 6 to 77 days, with the most frequent period being 14 days (7/25). Effects of different probiotics on different specific CNS functions in animals were analyzed and described in the following text.

\section{Anxiety}

Twelve studies tested anxiety-like behavior in animals (mice or rats). The anxiety-like behaviors were evaluated with the elevate plus/Barnes maze, light/dark box, defensive burying, open field/ arms, fear conditioning, and step-down tests. Three of them used a single strain of Bifidobacterium longum, all with positive results, ie, the animals showed less anxious behavior. ${ }^{22,35,36}$ Two studies using Lactobacillus helveticus also found reduced anxiety-like behaviors in immune-deficient mice and chronically restrained rats. ${ }^{29,37,38}$ Two studies used Lactobacillus rhamnosus but only one showed reduced anxiety behaviors. ${ }^{23,35}$ Two studies using Lactobacillus plantarum also found alleviated anxiety levels in mice after the intervention. ${ }^{20,21}$ Bifidobacterium breve and Lactobacillus fermentum were used once each and showed anxiolytic effects. ${ }^{30,36}$ Two studies using multi-strain probiotic combinations of $L$. rhamnosus $+L$. helveticus and B. longum + L. helveticus found reduced anxious behavior. $^{29,39}$

\section{Depression}

Nine studies focused on depression and all reported positive results except one. Depression-like behaviors were measured with the tail-suspension, forced-swim, and sucrose-preference tests. $B$. infantis was used twice but only one study found reduced depressionlike behavior. Each of the single strains of $B$. longum, $B$. breve, $L$. rhamnosus, and L. helveticus was also used once and all showed antidepressant effects. ${ }^{23,26,27,36,40}$ Two studies tested $L$. plantarum, but it only had an effect in mice with the early life stress of maternal separation. $^{20,21}$ One study used a multi-strain combination of $B$. longum + L. helveticus and also showed positive effects. ${ }^{41}$

\section{Cognitive function}

Eleven studies tested cognitive function, and all showed the probiotics to be beneficial for memory performance. Spatial memory was tested with the Morris water maze and the Barnes maze tests; other non-spatial memory abilities were measured with the novel object, fear conditioning, passive avoidance step-down, and T-maze tests.

Single strains of B. longum, B. breve, and L. helveticus were effective on both spatial and non-spatial memories. ${ }^{37,38,42,43}$ Single strains of $L$. fermentum and Clostridium butyricum improved spatial memory ability. ${ }^{30,37,42,44}$ Multi-strain probiotics that were assessed to be effective with regard to non-spatial memory included combinations of $L$. rhamnosus $+L$. helveticus ${ }^{29,45}$ and B. longum + L. helveticus, ${ }^{41}$ and combinations of Lactobacillus acidophilus + B. lactis + L. fermentum and L. plantarum + Lactobacillus curvatus in spatial memory. ${ }^{46,47}$

\section{Autism spectrum disorder and obsessive-compulsive disorder}

Autism spectrum disorder-related behaviors were tested with the open field and marble- burying tests for anxiety, the pre-pulse inhibition test for sensorimotor, ultrasonic vocalization for communicative, and the three-chamber social test for social interaction behaviors. Bifidobacterium fragilis improved behaviors related to the ASD in maternal immune activation mice, including anxietylike behavior, sensory gating and communicative behavior, but not social interaction behavior. ${ }^{31}$

Obsessive-compulsive disorder-related behaviors were also measured with the open field, marble burying, pre-pulse inhibition, ultrasonic vocalization and 3-chamber social tests. L. rhamnosus was found to be able to decrease obsessive-compulsive disorder-like behaviors in mice, but only locomotor ability and anxiety level. No effect was found in communicative or social interaction behaviors. ${ }^{48}$ However, a recent study investigated sickness behavior using a social investigative behavior paradigm, and found VSL\#3 improved sickness behavior with increased social exploratory behaviors. ${ }^{49}$

\section{Stress response}

Four studies involved stress induction to test behavioral response to stress. Stress was induced, with water avoidance stress as an acute stressor ${ }^{29,45,50}$ and maternal separation as a chronic stressor. ${ }^{27}$ Acute stress was used to induce anxiety, memory dysfunction and HPA response; chronic stress was used to induce depression.

Anxiety behavior was not successfully induced by water avoidance stress, while memory dysfunction was induced only in Gareau's study. ${ }^{45}$ A probiotic combination of L. rhamnosus $+L$. helveticus prevented non-spatial memory dysfunction induced by acute stress. ${ }^{45}$ One study only measured plasma corticosterone levels in response to acute stress and found a significant decrease due to Lactobacillus casei Shirota intervention. ${ }^{50}$ For behavioral changes caused by chronic stress exposure, $B$. infantis normalized depression-like behavior induced by maternal separation. ${ }^{27}$ 


\section{Mechanisms of action}

In addition to outcomes on behavioral levels, we also collected data at the physiological level, exploring endocrine, immune, neural chemical, and metabolic changes due to probiotics. Most of the studies investigated serum corticosteroid levels and found they were decreased by various probiotics: $L$. plantarum, $L$. helveticus, $L$. fermentum, L rhamnosus, and $L$. casei Shirota. ${ }^{20,23,30,37,44,45,50}$ Adrenocorticotropic hormone (ACTH) could also be decreased by $L$. helveticus and L. fermentum. ${ }^{30,38}$ Colon inflammation was alleviated and cytokine levels were influenced: inflammatory cytokines such as IL-6 and TNF- $\alpha$ were decreased and anti-inflammatory cytokines such as IL 10 were increased. ${ }^{20,30,35,37,38,40,42,45,47,49}$ These immune-effective probiotics were $L$. plantarum, $L$. helveticus, $L$. fermentum, L. acidophilus, B. longum, and L. rhamnosus. Brain monoamines, such as $5-\mathrm{HT}$ and DA, could be increased by the probiotics $L$. plantarum, $L$. helveticus, and B. infantis, while their metabolites were reduced. ${ }^{20,26,38}$ GABA receptor expression could be influenced by $L$. rhamnosus, depending on the brain area. ${ }^{23}$ Brain BDNF and c-Fos mRNA expression increased after probiotic intervention with $L$. helveticus, L. plantarum, L. rhamnosus, B. longum, and C. butyricum, while c-Fos in the hypothalamus paraventricular nucleus was decreased by $L$. casei Shirota. ${ }^{35,38,44,45,47}$ Two studies found effects of $L$. rhamnosus and B. longum that were mediated via the vagus nerve (ie, no effect in vagotomized mice), ${ }^{22,23}$ and one study found $L$. casei Shirota to enhance gastric vagal afferent activity. ${ }^{50}$ Enteric neuron excitability was inhibited by B. longum, ${ }^{22}$ while visceral sensitivity by colon distension was unaffected. ${ }^{43}$ One study found that a probiotic formulation of $B$. longum $+L$. helveticus reduced intestinal barrier permeability. ${ }^{31,41}$ Probiotics L. helveticus, $B$. infantis, and $B$. fragilis influenced metabolites by enhancing serum tryptophan levels and inhibiting its metabolites. ${ }^{26,31,42}$ Several studies conducted microbiota analyses on the fecal samples and found fecal microbiota were altered by probiotics: for example, Bacteroides and Lactobacillus were increased and Firmicutes decreased by $L$. fermentum. ${ }^{29-31,44,45}$ More details are shown in Table 1.

\section{Human Studies}

In total, 15 human studies were included. All of the selected studies had strong ratings in the quality assessment tool for quantitative studies, although one of the studies did not describe the age and gender of the participants in each group. ${ }^{51}$ Among the 15 studies, 8 used a single-strain probiotic (L. casei, L. casei subsp. rhamnosus, L. casei Shirota, L. plantarum, and B. infantis), of which 2 used probiotic containing milk, and the other 7 studies used multi- strain probiotics. Eight of the 15 studies found significant effects of the probiotic interventions. Doses of the effective interventions ranged from $10^{7}$ to $3.63 \times 10^{10}$, and the duration of the treatments ranged from 20 days to 8 weeks. Doses around $10^{9}(5 / 8)$ and $10^{10}$ (3/8) were used most often. Durations were most commonly 4 (6/15) and $8(4 / 15)$ weeks. Due to the heterogeneity of the studies (eg, probiotic interventions and measurements of CNS functions), we only describe the results based on the different interventions (Table 2).

\section{Psychiatric conditions}

Here, we summarize the studies on depression, anxiety, and/or mood together, because in most of the studies, questionnaires that tested multiple psychiatric conditions were used. Fifteen studies tested healthy participants with respect to anxiety, depression, distress levels, mood state, and behavior problems. ${ }^{39,51-62}$ The measurement tools included the General Health Questionnaire (GHQ), the Depression Anxiety and Stress Scale (DASS), the Leiden Index of Depression Sensitivity-Revised (LEIDS-r), the Positive and Negative Symptom Scale (PANSS), the State-Trait Anxiety Inventory (STAI), the Development Behavior Checklist (DBC), the Beck Depression Inventory (BDI), the Beck Anxiety Inventory (BAI), the Hopkins Symptom Checklist (HSCL-90), the Hospital Anxiety and Depression Scale (HADS), the Perceived Stress Scale (PSS), the Coping Checklist (CCL) (also used to counter the stress of daily life), and the questionnaire-based Profile of Mood State (POMS). Due to the different questionnaires, scales, and their combinations used in these studies, we only report here whether the probiotics treatment improved mental health/mood.

One study compared a probiotic capsule (containing Lactobacillus casei, L. acidophilus, L. rhamnosus, Lactobacillus bulgaricus, $B$. breve, B. longum, and Streptococcus thermophilus) and a probiotic yogurt (containing $B$. lactis and $L$. acidophilus) with the combination of conventional yogurt and a placebo capsule, and found the former two were more effective in alleviating distress, anxiety, and depression in petrochemical workers. ${ }^{52} \mathrm{~A}$ recent study using multi-strain probiotics found improvement in the LEIDS-r score, which is predictive of depression. ${ }^{53}$ Two studies by Messaoudi et $\mathrm{al}^{39,61}$ found probiotic formulations of $B$. longum and L. helveticus could improve anxiety and depression in all participants, and also in those who had lower urinary free cortisol levels at baseline. One study using $L$. casei Shirota-containing milk improved mood only in the bottom third of the depressed distribution at baseline. ${ }^{62} \mathrm{~A}$ study in chronic fatigue syndrome patients also used L. casei Shirota and found decreased anxiety levels following treatment. ${ }^{51}$ One 
Table 2. Studies of Probiotic Effects on Central Nervous System Functions in Humans

\begin{tabular}{|c|c|c|c|c|c|c|}
\hline Study & Participants & Probiotic & $\begin{array}{c}\text { Dosage } \\
\text { (CFU/day) } \\
\text { and duration }\end{array}$ & $\begin{array}{l}\text { CNS } \\
\text { function }\end{array}$ & Outcome & $\begin{array}{l}\text { Secondary } \\
\text { outcome }\end{array}$ \\
\hline $\begin{array}{l}\text { Takada et } \\
\mathrm{al}^{50} 2016\end{array}$ & $\begin{array}{l}140 \text { healthy } \\
\text { students }\end{array}$ & L. casei Shirota YIT 9029 & $\begin{array}{l}1 \times 10^{9} \\
8 \text { weeks }\end{array}$ & STAI & $\begin{array}{l}\text { No difference of } \\
\text { STAI score }\end{array}$ & $\begin{array}{l}\text { Change in salivary } \\
\text { cortisol level before } \\
\text { exam } \downarrow \\
\text { Decrease in physical } \\
\text { symptoms } \downarrow\end{array}$ \\
\hline $\begin{array}{l}\text { Moham- } \\
\text { madi et al, } \\
2015\end{array}$ & $\begin{array}{l}70 \text { petrochemical } \\
\text { workers }\end{array}$ & $\begin{array}{l}\text { probiotic yogurt } \\
\text { (L. acidophilus LA5 + } \\
\text { B. lactis Bb12) + } \\
\text { placebo capsule; } \\
\text { Conventional yogurt } \\
\text { (S. thermophilus and } \\
\text { L. bulgaricus.) + } \\
\text { probiotic capsule (L. casei, } \\
\text { L. acidophilus, L. rhamnosus, } \\
\text { L. bulgaricus, B. breve, } \\
\text { B. longum, S. thermophilus) }\end{array}$ & $\begin{array}{l}\text { probiotic yogurt: } \\
10^{7} \text { in total } \\
\text { Probiotic capsule: } 3 \\
\times 10^{3}, 3 \times 10^{7}, 7 \\
\times 10^{9}, 5 \times 10^{8}, 2 \\
\times 10^{10} ; 10^{9}, 3 \times \\
10^{8} \text {, respectively } \\
\left(2.88 \times 10^{10} \text { in }\right. \\
\text { total }) / 6 \text { weeks }\end{array}$ & $\begin{array}{l}\text { GHQ } \\
\text { DASS } \\
\text { HPA axis activity }\end{array}$ & $\begin{array}{l}\text { Improvement of } \\
\text { GHQ and DASS in } \\
\text { probiotics yogurt and } \\
\text { probiotics capsule } \\
\text { groups; no difference } \\
\text { in HPA axis activity }\end{array}$ & NA \\
\hline $\begin{array}{l}\text { Steenbergen } \\
\text { et al, }{ }^{53} 2015\end{array}$ & $\begin{array}{l}40 \text { healthy } \\
\text { volunteers }\end{array}$ & $\begin{array}{l}\text { Ecologic Barrier: } \\
\text { B. bifidum W23, B. lactis } \\
\text { W52, L. acidophilus W37, } \\
\text { L. brevis W63, L. casei } \\
\text { W56, L. salivarius W24, } \\
\text { Lactococcus lactis } \\
\text { (W19 and W58). }\end{array}$ & $\begin{array}{l}5 \times 10^{9} \\
4 \text { weeks }\end{array}$ & $\begin{array}{l}\text { LEIDS-r } \\
\text { BDI } \\
\text { BAI }\end{array}$ & $\begin{array}{l}\text { Improvement of total } \\
\text { score and item } \\
\text { 'rumination' of } \\
\text { LEIDS-r. } \\
\text { No difference of scores } \\
\text { of Beck Depression } \\
\text { and Beck Anxiety } \\
\text { Inventory }\end{array}$ & $\mathrm{NA}$ \\
\hline $\begin{array}{l}\text { Dickerson et } \\
\mathrm{al}^{54} 2014\end{array}$ & $\begin{array}{l}65 \text { schizophrenia } \\
\text { patients }\end{array}$ & $\begin{array}{l}\text { L. rhamnosus GG } \\
\text { (ATCC 53103+ } \\
\text { B. animalis subsp. lactis Bb12 }\end{array}$ & $\begin{array}{l}2 \times 10^{9} \\
14 \text { weeks }\end{array}$ & PANSS & $\begin{array}{l}\text { No difference of toll } \\
\text { score or positive, } \\
\text { negative or general } \\
\text { scores. }\end{array}$ & $\begin{array}{l}\text { Severe difficulty in } \\
\text { bowel movement } \downarrow\end{array}$ \\
\hline $\begin{array}{l}\text { Vaghef- } \\
\text { Mehrabany } \\
\text { et al, }{ }^{55} 2014\end{array}$ & $\begin{array}{l}46 \text { patients with } \\
\text { rheumatoid } \\
\text { arthritis }\end{array}$ & L. casei 01 & $\begin{array}{l}10^{8} \\
8 \text { weeks }\end{array}$ & STAI & $\begin{array}{l}\text { No difference of } \\
\text { STAI score }\end{array}$ & $\begin{array}{l}\text { Dietary: NA } \\
\text { Cytokines: pro } \\
\text { inflammatory } \\
\text { cytokine TNF- } \alpha \text {, } \\
\text { IL-6, and IL-12 } \downarrow \text {, } \\
\text { anti-inflammatory } \\
\text { cytokine IL-10 } \uparrow\end{array}$ \\
\hline $\begin{array}{l}\text { Dapoigny et } \\
\mathrm{al}^{56}{ }^{56} 2012\end{array}$ & 50 IBS patients & $\begin{array}{l}\text { L. casei subsp. rhamnosus } \\
\text { LCR35 }\end{array}$ & $\begin{array}{l}6 \times 10^{8} \\
4 \text { weeks }\end{array}$ & HADS & $\begin{array}{l}\text { No difference in } \\
\text { HADS score }\end{array}$ & $\begin{array}{l}\text { IBS severity score: } \\
\text { only clinically } \\
\text { relevant decreased } \\
\text { in subtype IBS-D } \downarrow \\
\text { Presence of } \\
\text { Lactobacillus in } \\
\text { feces: } 85 \% \text { of patients }\end{array}$ \\
\hline $\begin{array}{l}\text { Simrén et } \\
\mathrm{al}^{57} 2010\end{array}$ & 74 IBS patients & $\begin{array}{l}\text { Milk fermented with yoghurt } \\
\text { bacteria } L \text {. bulgaricus }+S \text {. } \\
\text { thermophilus and containing } \\
\text { L. paracasei F19 + } \\
\text { L. acidophilus La5 }+ \\
\text { B. lactis Bb12 }\end{array}$ & $\begin{array}{l}2 \times 10^{10} \\
8 \text { weeks }\end{array}$ & HADS & $\begin{array}{l}\text { No difference of } \\
\text { HADS score }\end{array}$ & $\begin{array}{l}\text { Diet: same among } \\
\text { groups }\end{array}$ \\
\hline $\begin{array}{l}\text { Whorwell et } \\
\mathrm{al}^{58} 2006\end{array}$ & $\begin{array}{l}362 \text { female IBS } \\
\text { patients }\end{array}$ & B. infantis 35624 & $\begin{array}{l}10^{6}, 10^{8}, 10^{10} \\
4 \text { weeks }\end{array}$ & HADS & $\begin{array}{l}\text { No difference in any } \\
\text { of the dosages }\end{array}$ & $\begin{array}{l}\text { IBS symptom: } \downarrow \\
\text { in } 10^{8} \text { group }\end{array}$ \\
\hline
\end{tabular}


Table 2. Continued

\begin{tabular}{|c|c|c|c|c|c|c|}
\hline Study & Participants & Probiotic & $\begin{array}{c}\text { Dosage } \\
\text { (CFU/day) } \\
\text { and duration }\end{array}$ & $\begin{array}{l}\text { CNS } \\
\text { function }\end{array}$ & Outcome & $\begin{array}{l}\text { Secondary } \\
\text { outcome }\end{array}$ \\
\hline $\begin{array}{l}\text { Reale et al, } \\
2012\end{array}$ & 72 male smokers & L. casei Shirota & $\begin{array}{l}4 \times 10^{10} \\
3 \text { weeks }\end{array}$ & STAI & $\begin{array}{l}\text { No difference in } \\
\text { STAI score }\end{array}$ & $\begin{array}{l}\text { Natural killer cell } \\
\text { activity } \uparrow \\
\text { CD16+ cell } \uparrow \\
\text { BMI: NA } \\
\text { Bowel function } \uparrow\end{array}$ \\
\hline $\begin{array}{l}\text { Parracho et } \\
\text { al, }^{60} 2010\end{array}$ & $\begin{array}{l}15 \text { children } \\
(4-16 Y) \text { with } \\
\text { ASD }\end{array}$ & L. plantarum WCFS1 & $\begin{array}{l}4.5 \times 10^{10} \\
3 \text { weeks }\end{array}$ & $\mathrm{DBC}$ & $\begin{array}{l}\text { No significant } \\
\text { difference in DBC } \\
\text { score }\end{array}$ & $\begin{array}{l}\text { Bowel function: only } \\
\text { different in stool } \\
\text { consistency } \\
\text { Fecal microbiota: } \\
\text { Lactobacillus Lab } \\
158 \uparrow, \text { Clostridium } \\
\text { Erec } 482 \downarrow\end{array}$ \\
\hline $\begin{array}{l}\text { Messaoudi } \\
\text { et al, }{ }^{39} 2011\end{array}$ & $\begin{array}{l}55 \text { healthy } \\
\text { volunteers }\end{array}$ & $\begin{array}{l}\text { L. helveticus R0052 + } \\
\text { B. longum R0175 }\end{array}$ & $\begin{array}{l}3 \times 10^{9} \\
4 \text { weeks }\end{array}$ & $\begin{array}{l}\text { HSCL-90 } \\
\text { HADS } \\
\text { PSS } \\
\text { CCL }\end{array}$ & $\begin{array}{l}\text { Improvement of } \\
\text { anxiety, depression } \\
\text { and problem solving, } \\
\text { and reduced UFC } \\
\text { level in probiotics } \\
\text { group }\end{array}$ & $\begin{array}{l}\text { Median urinary free } \\
\text { cortisol } \downarrow\end{array}$ \\
\hline $\begin{array}{l}\text { Messaoudi } \\
\text { et al, }^{61} 2011\end{array}$ & $\begin{array}{l}25 \text { healthy } \\
\text { volunteers (with } \\
\text { lower UFC levels } \\
\text { than median } \\
\text { value at baseline }\end{array}$ & $\begin{array}{l}\text { L. helveticus R0052 + } \\
\text { B. longum R0175 }\end{array}$ & $\begin{array}{l}3 \times 10^{9} \\
4 \text { weeks }\end{array}$ & $\begin{array}{l}\text { HSCL-90 } \\
\text { HADS } \\
\text { PSS } \\
\text { CCL }\end{array}$ & $\begin{array}{l}\text { Improvement of } \\
\text { anxiety and depression } \\
\text { in probiotics group }\end{array}$ & NA \\
\hline $\begin{array}{l}\text { Rao et al, } \\
2009\end{array}$ & 35 CFS patients & L. casei Shirota & $\begin{array}{l}2.4 \times 10^{10} \\
8 \text { weeks }\end{array}$ & $\begin{array}{l}\mathrm{BDI} \\
\mathrm{BAI}\end{array}$ & $\begin{array}{l}\text { Decreased anxiety } \\
\text { symptoms in probiotic } \\
\text { group }\end{array}$ & $\begin{array}{l}\text { Fecal microbiota: } \\
\text { aerobes } \uparrow \text {, anaerobes } \\
\uparrow \text { Bifidobacteria } \uparrow \text {, } \\
\text { Lactobacillus } \uparrow\end{array}$ \\
\hline $\begin{array}{l}\text { Benton et } \\
\mathrm{al}^{62} 2007\end{array}$ & $\begin{array}{l}124 \text { healthy } \\
\text { volunteers }\end{array}$ & $\begin{array}{l}\text { L. casei Shirota } \\
\text { (containing milk) }\end{array}$ & $\begin{array}{l}6.5 \times 10^{9} \\
10 / 20 \text { days }\end{array}$ & $\begin{array}{l}\text { Questionnaire-based } \\
\text { POMS } \\
\text { Episodic memory } \\
\text { (Wechsler Memory } \\
\text { Scale) } \\
\text { Retrieval from } \\
\text { long-term memory } \\
\text { Verbal fluency } \\
\text { Eating-associated } \\
\text { behavior } \\
\text { NART }\end{array}$ & $\begin{array}{l}\text { Improved mood in the } \\
\text { bottom third of the } \\
\text { POMS depressed/ } \\
\text { elated distribution at } \\
\text { baseline in probiotics } \\
\text { group after } 20 \text { days } \\
\text { Improved memory in } \\
\text { probiotics group after } \\
20 \text { days }\end{array}$ & NA \\
\hline $\begin{array}{l}\text { Tillisch et } \\
\mathrm{al}^{63} 2013\end{array}$ & 36 healthy females & $\begin{array}{l}\text { FMPP (B. lactis I-2494 } \\
{[\mathrm{DN}-173 \text { 010], containing }} \\
\text { yogurt starters include } S \text {. } \\
\text { thermophilus I-1630, } \\
\text { L. bulgaricus I-1632 and } \\
\text { I-1519) and Lactococcus } \\
\text { lactis subsp lactis I-1631 }\end{array}$ & $\begin{array}{l}\text { B. lactis : } \\
1.25 \times 10^{10} \\
\text { S. thermophilus } \\
\text { and } L . \text { bulgaricus : } \\
1.2 \times 10^{9} \\
4 \text { weeks }\end{array}$ & $\begin{array}{l}\text { Standard emotional } \\
\text { faces-attention task } \\
\text { for } \mathrm{MMRI}\end{array}$ & $\begin{array}{l}\text { Decreased activity to } \\
\text { emotional faces in a } \\
\text { large distributed } \\
\text { network } \\
\text { Changes in midbrain } \\
\text { connectivity during } \\
\text { resting state }\end{array}$ & NA \\
\hline
\end{tabular}

STAI, State-Trait Anxiety Inventory; NA, not applicable; GHQ, General Health Questionnaire; DASS, Depression Anxiety and Stress Scale; HPA, hypothalamicpituitary-adrenal; BMI, body mass index; LEIDS-r, Leiden Index of Depression Sensitivity-Revised; BDI, Beck Depression Inventory; BAI, Beck Anxiety Inventory; PANSS, Positive and Negative Symptom Scale; IBS, irritable bowel syndrome; HADS, Hospital Anxiety and Depression Scale; ASD, autism spectrum disorder; IBS-D, diarrhea-predominant IBS; DBC, Development Behavior Checklist; HSCL-90, Hopkins Symptom Checklist; PSS, Perceived Stress Scale; CCL, Coping Checklist; UFC, urinary free cortisol; CFS, chronic fatigue syndrome; POMS, questionnaire-based Profile of Mood State; NART, National Adult Reading Rest; FMPP, fermented milk product with probiotic; fMRI, functional magnetic resonance imaging; $B$. animalis, Bifidobacterium animalis; $B$. breve, Bifidobacterium breve; $B$. bifidum, Bifidobacterium bifidum; B. infantis, Bifidobacterium infantis; $B$. lactis, Bifidobacterium lactis; B. longum, Bifidobacterium longum; $L$. acidophilus, Lactobacillus acidophilus; L. brevis, Lactobacillus brevis; L. bulgaricus, Lactobacillus bulgaricus; L. casei, Lactobacillus casei; L. helveticus, Lactobacillus helveticus; L. paracasei, Lactobacillus paracasei; L. plantarum, Lactobacillus plantarum; L. rhamnosus, Lactobacillus rhamnosus; L. salivarius, Lactobacillus salivarius; $S$. thermophiles, Streptococcus thermophiles. 
recent study, also using $L$. casei Shirota, found decreased salivary cortisol levels in university students in response to stress, although no significant difference in STAI score was observed. ${ }^{50}$

However, other studies found no significant effect of their probiotic interventions. Patients with schizophrenia showed no change in PANSS score after treatment with $L$. rhamnosus for 14 weeks. ${ }^{54}$ Patients with rheumatoid arthritis showed no change in anxiety levels, as assessed with the STAI after 8 weeks of $L$. casei. ${ }^{55}$ Three studies conducted in IBS patients all looked into HADS scores before and after interventions, but found no effect of $L$. casei or fermented milk with $L$. paracasei and $L$. acidophilus. ${ }^{56-58}$ In healthy male smokers, a 3-week intervention with $L$. casei showed no effect on STAI score. ${ }^{59}$ In children with ASD, a 3-week intervention with L. plantarum did not change the DBC score. ${ }^{60}$

\section{Memory and other cognitive abilities}

The study of Benton et $\mathrm{al}^{62}$ measured different memory and cognitive abilities in healthy participants, including episodic memory, tested with the Wechsler Memory Scale, retrieval from long-term memory, verbal fluency, eating-associated behavior, and premorbid intelligence, tested with the National Adult Reading Test. However, L. casei Shirota decreased memory abilities in all participants compared with the placebo, and had no effect on verbal fluency or eating-associated behavior.

\section{Neuroimaging study}

There was only one neuroimaging study, using functional magnetic resonance imaging ( $\mathrm{fMRI}$ ), investigating the change in brain activity to emotional stimuli and basal brain activation after intake of a fermented milk product with probiotic (FMPP) containing $B$. lactis with yogurt starters, $S$. thermophilus, L. bulgaricus, and Lactococcus lactis subsp. lactis. ${ }^{63}$ The FMPP decreased activity of a large distributed network including affective, viscerosensory, and somatosensory cortices to emotional faces, and changed midbrain connectivity during the resting state.

\section{Mechanisms of action}

Two studies found reduced cortisol levels in saliva and urine after probiotic interventions with $L$. casei Shirota and multi-strain $L$. helveticus $+B$. longum, respectively. ${ }^{39,50}$ The immune system could be improved by the probiotic $L$. casei, with evidence of reduced pro-inflammatory cytokines (TNF- $\alpha$, IL-6, and IL-12), increased regulatory cytokines (eg, IL-10), and increased natural killer cell activity in smokers. ${ }^{55,59}$ Only one study in humans investigated metabolites of the tryptophan pathway but did not find any significant change by probiotics. ${ }^{52}$ Many of the human studies looked at bowel function, and they did find reduced difficulties in bowel movement, IBS severity, and symptoms in patients. ${ }^{54,56,58,60}$ Microbiota analysis helped to confirm that fecal microbiota were altered by probiotic intervention: Lactobacillus was increased and Clostridium decreased by L. plantarum, whereas Bifidobacteria and Lactobacillus were increased by L. casei Shirota. ${ }^{51,60}$ More details are shown in Table 2.

Summarizing all the studies in animals and humans that focused on CNS functions, including psychiatry conditions (anxiety and depression) and memory abilities, Bifidobacterium and Lactobacillus were the probiotics used most frequently. Doses ranged from $10^{7}$ to $4 \times 10^{10} \mathrm{CFU}$ per day and most studies used $10^{9}$ and $10^{10} \mathrm{CFU}$ in animals and $3 \times 10^{9} \mathrm{CFU}$ in humans. The duration of intake ranged from 1 week to 6 months with the most frequent durations of 2 weeks in animals and 4 weeks in humans.

\section{Discussion}

The number of studies using probiotics to improve central nervous system function has increased over the past 10 years, though with a focus on effects in animals. Researchers have used various strains of probiotics and studied various CNS functions. Summarizing the divergent findings motivated us to perform a systematic review of this research area. Previously, there was no systematic review or meta-analysis of the effects of probiotics on CNS function in animals and humans. To date, there are a few reviews on probiotic effects on infantile colic, which may reflect peripheral nervous system action, and one recent study reviewing only human studies. ${ }^{64-66}$ Similarly, among the 56 RCTs so far which tested probiotics in adults with IBS that have or have not shown effects on peripheral (bowel) functions, ${ }^{15}$ none have investigated whether any CNS effect was affected or improved. We identified studies applying probiotics as single- or multiple-strain preparations in animals and humans. Because of the diversity of the interventions and the CNS functions tested in these studies, we did not perform a meta-analysis.

\section{Effects of Probiotics}

Combining all the studies in animals and humans, probiotics appear to have a positive effect in improving central nervous system function. However, a publication bias toward positive results cannot be excluded. Based on currently available studies, we can see that most of the studies used Bifidobacterium and Lactobacillus preparations, and most of them were effective in improving specific CNS functions. Again, however, negative results in relation to other functions may have been unreported, even in otherwise positive 
studies. Doses of $10^{9}$ and $10^{10} \mathrm{CFU}$ have been used in most studies showing an effect on behaviors. An intake of the probiotic for 2 weeks in animals and 4 weeks in humans is apparently sufficient to elicit measurable effects.

$B$. longum, $B$. breve, $B$. infantis, $L$. helveticus, $L$. rhamnosus, $L$. plantarum, and $L$. casei were the most commonly used preparations in these studies, as single- or multi-strain preparations, all of which were able to improve anxiety, depression, and memory related behaviors, based on animal models. All of these probiotics are regarded as "good" bacteria, presumably inhibiting the growth of harmful bacteria or pathogens and/or improving the immune system. ${ }^{67-69}$ These probiotics were also found to reduce the symptoms of gastrointestinal disease, such as irritable bowel syndrome. ${ }^{15,16,58,70}$ Probiotics may play an important role in gut-brain axis communication, thereby benefiting both the brain and the gut.

While some studies found no significant effect of probiotic intervention, the evidence is inadequate to conclude that the interventions were ineffective because some difficulties and/or weak points exist. For example, schizophrenia as a severe mental illness, and being closely related to a genetic disposition, may be a case in which probiotics can hardly be expected to have a significant effect on changing symptoms. ${ }^{54}$ Probiotic doses in some studies were below the supposed effective dosages (at least $10^{9} \mathrm{CFU}$ ), such as $10^{8}$ $\mathrm{CFU}$ in the study in rheumatoid arthritis patients and $10^{6}, 5 \times 10^{7}$, and $10^{8} \mathrm{CFU}$ in IBS patients. ${ }^{55-58}$ Also, in two studies, one in male smokers and one in children with ASD, the intervention periods were 3 weeks, shorter than the effective period, which seems to be 4 weeks, that can make a measurable effect. ${ }^{59,60}$

Also, caution is warranted when drawing conclusions from the human studies that used psychological questionnaires and/or scales rather than behavioral or neuropsychological experiments, resulting in subjective biases. The clinical efficacy of probiotic interventions and guidelines for their administration in diseases such as diarrhea, allergies, IBS, and inflammatory bowel disease has been addressed in previous reviews. ${ }^{15,71,72}$ More studies investigating probiotic effects in mental disorders are needed.

\section{Mechanisms of Action of Probiotic Effects}

The current state-of-the-art suggests several mechanisms: the endocrine system, immune system, action of enteric neurons, and commensal bacteria (or their metabolic activity). This evidence has come primarily from preclinical studies, while a few clinical studies have analyzed cortisol and cytokine levels in saliva and plasma. The HPA axis activity has been linked closely to mood disorders and memory abilities. ${ }^{737}$ Many probiotics reduced HPA axis ac- tivity by decreasing CORT and/or ACTH levels, including most of the Lactobacillus strains tested: $L$. plantarum, $L$. helveticus, $L$. fermentum, $L$. rhamnosus, and $L$. casei. ${ }^{19,22,29,36,39,43,44,49}$ However, single strains of Bifidobacterium such as $B$. infantis, $B$. longum, and $B$. breve had no effect on CORT levels. ${ }^{27,29,36} \mathrm{BDNF}$ is the key for neurogenesis and synaptic plasticity, which structurally support CNS function. ${ }^{75,76}$ Lactobacillus (L. plantarum, L. helveticus, $L$. fermentum), Bifidobacterium (B. longum), and $C$. butyricum increased brain BDNF. ${ }^{34,37,43,46}$ Neuronal activation can be indicated by the nuclear localization of c-Fos; the effect of c Fos in the CNS depends on its location. The combination of $L$. rhamnosus $+L$. helveticus improved c Fos expression in the hippocampus and improved memory ability, while $L$. casei decreased it in the paraventricular nucleus of hypothalamus region and alleviated stress responses. ${ }^{45,50}$ Neurotransmitters 5-HT, DA, and GABA are closely related to psychiatric conditions, and were influenced directly by many strains of probiotic ( $L$. plantarum, $L$. helveticus, $L$. fermentum, $L$. rhamnosus, and $B$. infantis). The vagus nerve has been proposed as a pathway of probiotic effects because neurochemical and behavioral changes due to $L$. rhamnosus and $B$. longum were not seen in vagotomized animals. Direct evidence for the role of the vagus nerve also comes from studies showing that gastric vagal afferent activity was enhanced by $L$. casei. The excitability of the enteric nervous system, which is connected to the vagus nerve, has been shown to be modulated by $B$. longum. ${ }^{22}$

Probiotics also alter CNS function indirectly through several other pathways. $L$. helveticus, $B$. infantis, and $B$. fragilis enhanced serum tryptophan (precursor of 5-HT) levels and reduced its metabolites. Most of the probiotics tested affected the immune system by decreasing pro-inflammatory cytokines and increasing antiinflammatory cytokines. Another important pathway through which probiotics may modulate CNS function is intestinal barrier permeability, which is essential for maintaining the immune and nervous systems. Increased intestinal barrier permeability is associated with psychiatric disorders, such as depression and autism, while it can be restored by probiotic formulations of $B$. longum and $L$. helveticus, along with improved CNS function. ${ }^{31,41,77}$

According to the data reviewed, different probiotics exhibited several common effects; however, these effects were strain-dependent and occurred via different pathways at a lower level of the CNS. Thus, more studies are needed for clarify which probiotics target which central biochemical substances and behaviors. In clinical applications, interventions with a probiotics cocktail may have greater effects, because different probiotics may create their effects at the same time through different pathways. However, as yet, such 
an approach currently lacks clinical evidence. ${ }^{15}$

\section{Translation of Animal Studies}

There are many animal studies about the gut microbiome-brain interaction using germ-free, specific pathogen free (SPF), or gnotobiotic animals, colonization with specific microbiota, probiotic intake, and infections to deliberately alter the GM and to manipulate CNS function. ${ }^{9,29,30,35,37,45,78-82}$ In humans, we are not able to adapt most of these models for ethical reasons. Ten of the animal studies included in our review used probiotics in animals whose health state had been disturbed by various manipulations, which included antibiotic treatment, gene knockout, inflammation, infection, maternal immune activation, hyperammonemia, and diabetic induction, and depression induced by myocardial infarction. All of the manipulations were aimed at inducing changes in CNS function, including anxiety and depression-like behavior, memory impairment, or ASD-like behaviors. In humans, interfering with the participants' healthy state is not an option. What is possible is to explore the GM composition, correlating it to certain behaviors, and using probiotics to manipulate the GM-brain interaction. It is also possible to temporarily affect single functions, such as the stress response at the central level or the GM composition by varying the food or drugs used. As yet, evidence from studies using probiotics is confined to animal studies. Validity estimates of probiotic intervention from human studies are still missing and thus need to be carried out.

The translation of behavioral models from animals to humans has both possibilities and limitations. The tests used to measure anxiety in animals, such as the elevate plus/Barnes maze, light/ dark box, defensive burying, open field/arms, and step-down, have no equivalents in humans, and neither do tests such as the forced swim and maternal separation for inducing depression and negative mood. In human studies, measurements of anxiety and depression rely primarily on scales such as the HADS, which has accuracy issues due to subjectivity and emotional bias from the participants/patients. Moreover, it remains questionable whether the behavioral tests used in animals do, in fact, adequately reflect the assumed CNS dysfunction (anxiety, depression) in humans and, more specifically, in patients. This leads to a demand for appropriate behavioral tests of anxiety and depression not only for patients with psychiatric disorders but also for the healthy population, and for adequate behavioral measures in animals that match these functions and dysfunctions in humans.

In addition to behavioral measurements, neuroimaging methods may provide insights as to what is altered in the brain that causes behavioral changes after the consumption of probiotics. An emotional faces attention task used in the fMRI study of Tillisch et $\mathrm{al}^{63}$ is one example: the brain response to emotional stimuli that may be related to psychiatric conditions was changed after a 4-week intake of probiotics.

Learning and memory abilities can be tested via numerous paradigms in humans. For spatial memory, there are computerized versions of the Morris water maze (VMWT) and the Blue Velvet Arena (BVA), which is also a variant of the Morris water maze for humans. ${ }^{83-86}$ For non-spatial memory, object recognition tasks and fear conditioning have been used widely in humans. These memory tasks can be conducted in combination with neuroimaging experiments, such as fMRI and magnetoencephalography (MEG) experiments.

There are also several ways to experimentally induce stress in humans. The Trier Social Stress Test, developed 20 years ago, during which participants are asked to play a role in a job interview, or in performing a public speech, can effectively increase the HPA axis and sympathetic-adrenal-medullary activity. ${ }^{87}$ Social stress can also be induced using the Cyberball paradigm, during which stress comes from social exclusion and/or ostracism. ${ }^{88,89}$ Noise as a stressor is easy to manipulate in a laboratory environment by exposing participants to unpleasant sounds so as to induce psychological stress. Cognitive tasks can also stimulate stress responses with the advantage of being able to study the stress level by measuring task performance. ${ }^{90}$ Other and more physical stressors include cold pressor tasks, ${ }^{91}$ heat pain, ${ }^{92}$ and the $\mathrm{CO}_{2}$ challenge test, inducing stress/ panic in participants by inhaling carbon dioxide-enriched air. ${ }^{93}$ All of these have also been shown to be compatible with brain imaging studies.

\section{Conclusions and Indications}

We reviewed the effect of probiotics on the central nervous system in randomized controlled trials in animals and humans, and analyzed the possibility of translating animal models to human studies because few human studies have been conducted to date. According to the qualitative analyses of current studies, we can provisionally draw the conclusion that $B$. longum, $B$. breve, $B$. infantis, L. helveticus, L. rhamnosus, L. plantarum, and L. casei were most effective in improving CNS function, including psychiatric diseaseassociated functions (anxiety, depression, mood, stress response) and memory abilities. Doses between $10^{9}$ and $10^{10} \mathrm{CFU}$ and durations of 2 weeks in animals and 4 weeks in humans have shown sufficient effects. Also, translations of animal studies to human studies may be applicable. Human studies can be conducted using the same probiotics and similar experimental paradigms in the emotional and 
neurocognitive domains. More experimental designs in humans should be developed, and more neuroimaging studies should be conducted rather than using only psychological questionnaires or scales. In addition to studies in healthy populations, clinical studies in patients with mental diseases would be worthwhile, because those with gastrointestinal disorders and psychiatric comorbidities, in general, appear to benefit from probiotic interventions.

Financial support: The research leading to these results has received funding from the People Programme of the European Union's Seventh Framework Programme under REA grant agreement No. 607652 (NeuroGut).

\section{Conflicts of interest: None.}

Author contributions: Paul Enck conceptualized the paper; Huiying Wang reviewed and evaluated the literature; and Huiying Wang, In-Seon Lee, Christoph Braun, and Paul Enck wrote the manuscript.

\section{References}

1. Ley RE, Peterson DA, Gordon JI. Ecological and evolutionary forces shaping microbial diversity in the human intestine. Cell 2006;124:837848.

2. Gill SR, Pop M, Deboy RT, et al. Metagenomic analysis of the human distal gut microbiome. Science 2006;312:1355-1359.

3. Farmer AD, Randall HA, Aziz Q. It's a gut feeling: how the gut microbiota affects the state of mind. J Physiol 2014;592:2981-2988.

4. Keightley PC, Koloski NA, Talley NJ. Pathways in gut-brain communication: evidence for distinct gut-to-brain and brain-to-gut syndromes. Aust N Z J Psychiatry 2015;49:207-214.

5. Dinan TG, Stanton C, Cryan JF. Psychobiotics: a novel class of psychotropic. Biol Psychiatry 2013;74:720-726.

6. Collins SM, Surette M, Bercik P. The interplay between the intestinal microbiota and the brain. Nat Rev Microbiol 2012;10:735-742.

7. Cryan JF, O'Mahony SM. The microbiome-gut-brain axis: from bowel to behavior. Neurogastroenterol Motil 2011;23:187-192.

8. Cryan JF, Dinan TG. Mind-altering microorganisms: the impact of the gut microbiota on brain and behaviour. Nat Rev Neurosci 2012;13:701712.

9. Sudo N, Chida Y, Aiba Y, et al. Postnatal microbial colonization programs the hypothalamic-pituitary-adrenal system for stress response in mice. J Physiol 2004;558(Pt 1):263-275.

10. Tomova A, Husarova V, Lakatosova $S$, et al. Gastrointestinal microbiota in children with autism in Slovakia. Physiol Behav 2015;138:179-187.

11. Kang DW, Park JG, Ilhan ZE, et al. Reduced incidence of Prevotella and other fermenters in intestinal microflora of autistic children. PLoS One 2013;8:e68322.

12. Scheperjans F, Aho V, Pereira PA, et al. Gut microbiota are related to Parkinson's disease and clinical phenotype. Mov Disord 2015;30:350-358.

13. Rijkers GT, de Vos WM, Brummer RJ, Morelli L, Corthier G, Marteau $\mathrm{P}$. Health benefits and health claims of probiotics: bridging science and marketing. Br J Nutr 2011;106:1291-1296.

14. Lilly DM, Stillwell RH. Probiotics: growth-promoting factors produced by microorganisms. Science 1965;147:747-748.

15. Mazurak N, Broelz E, Storr M, Enck P. Probiotic therapy of the irritable bowel syndrome: why is the evidence still poor and what can be done about it? J Neurogastroenterol Motil 2015;21:471-485.

16. Ford AC, Quigley EM, Lacy BE, et al. Efficacy of prebiotics, probiotics, and synbiotics in irritable bowel syndrome and chronic idiopathic constipation: systematic review and meta-analysis. Am J Gastroenterol 2014;109:1547-1561.

17. Lee BJ, Bak YT. Irritable bowel syndrome, gut microbiota and probiotics. J Neurogastroenterol Motil 2011;17:252-266.

18. DuPont HL. Biologic properties and clinical uses of rifaximin. Expert Opin Pharmacother 2011;12:293-302.

19. Chichlowski M, Rudolph C. Visceral pain and gastrointestinal microbiome. J Neurogastroenterol Motil 2015;21:172-181.

20. Liu YW, Liu WH, Wu CC, et al. Psychotropic effects of Lactobacillus plantarum PS128 in early life-stressed and naïve adult mice. Brain Res 2016;1631:1-12.

21. Liu WH, Chuang HL, Huang YT, et al. Alteration of behavior and monoamine levels attributable to Lactobacillus plantarum PS128 in germ-free mice. Behav Brain Res 2016;298:202-209.

22. Bercik P, Park AJ, Sinclair D, et al. The anxiolytic effect of Bifidobacterium longum NCC3001 involves vagal pathways for gut-brain communication. Neurogastroenterol Motil 2011;23:1132-1139.

23. Bravo JA, Forsythe P, Chew MV, et al. Ingestion of Lactobacillus strain regulates emotional behavior and central GABA receptor expression in a mouse via the vagus nerve. Proc Natl Acad Sci USA 2011;108:1605016055.

24. O'Sullivan E, Barrett E, Grenham S, et al. BDNF expression in the hippocampus of maternally separated rats: does Bifidobacterium breve 6330 alter BDNF levels? Benef Microbes 2011;2:199-207.

25. Ait-Belgnaoui A, Durand H, Cartier C, et al. Prevention of gut leakiness by a probiotic treatment leads to attenuated HPA response to an acute psychological stress in rats. Psychoneuroendocrinology 2012;37:18851895.

26. Desbonnet L, Garrett L, Clarke G, Bienenstock J, Dinan TG. The probiotic Bifidobacteria infantis: an assessment of potential antidepressant properties in the rat. J Psychiatr Res 2008;43:164-174.

27. Desbonnet L, Garrett L, Clarke G, Kiely B, Cryan JF, Dinan TG. Effects of the probiotic Bifidobacterium infantis in the maternal separation model of depression. Neuroscience 2010;170:1179-1188.

28. Kwok LY, Wang L, Zhang J, Guo Z, Zhang H. A pilot study on the effect of Lactobacillus casei Zhang on intestinal microbiota parameters in Chinese subjects of different age. Benef Microbes 2014;5:295-304

29. Smith CJ, Emge JR, Berzins K, et al. Probiotics normalize the gut-brainmicrobiota axis in immunodeficient mice. Am J Physiol Gastrointest 
Liver Physiol 2014;307:G793-G802.

30. Wang T, Hu X, Liang S, et al. Lactobacillus fermentum NS9 restores the antibiotic induced physiological and psychological abnormalities in rats. Benef Microbes 2015;6:707-717.

31. Hsiao EY, McBride SW, Hsien S, et al. Microbiota modulate behavioral and physiological abnormalities associated with neurodevelopmental disorders. Cell 2013;155:1451-1463.

32. Moher D, Shamseer L, Clarke M, et al. Preferred reporting items for systematic review and meta-analysis protocols (PRISMA-P) 2015 statement. Syst Rev 2015;4:2046-4053.

33. Higgins JPT, Green S. Cochrane handbook for systematic reviews of interventions. Version 5.1.0. The Cochrane Collaboration, 2011. Available from URL: http://handbook.cochrane.org/ (accessed 22 Sep 2016).

34. National Collaborating Centre for Methods and Tools (2008). Quality assessment tool for quantitative studies. Hamilton: MaMaster University (updated 13 April 2010). Available from URL: http://www.nccmt.ca/ resources/search/14 (accessed 22 Sep 2016).

35. Bercik P, Verdu EF, Foster JA, et al. Chronic gastrointestinal inflammation induces anxiety-like behavior and alters central nervous system biochemistry in mice. Gastroenterology 2010;139:2102-2112, e1.

36. Savignac HM, Kiely B, Dinan TG, Cryan JF. Bifidobacteria exert strainspecific effects on stress-related behavior and physiology in BALB/c mice. Neurogastroenterol Motil 2014;26:1615-1627.

37. Ohland CL, Kish L, Bell H, et al. Effects of Lactobacillus helveticus on murine behavior are dependent on diet and genotype and correlate with alterations in the gut microbiome. Psychoneuroendocrinology 2013;38:1738-1747.

38. Liang S, Wang T, Hu X, et al. Administration of Lactobacillus helveticus NS8 improves behavioral, cognitive, and biochemical aberrations caused by chronic restraint stress. Neuroscience 2015;310:561-577.

39. Messaoudi M, Lalonde R, Violle N, et al. Assessment of psychotropiclike properties of a probiotic formulation (Lactobacillus helveticus R0052 and Bifidobacterium longum R0175) in rats and human subjects. Br J Nutr 2011;105:755-764.

40. Singh PK, Chopra K, Kuhad A, Kaur IP. Role of Lactobacillus acidophilus loaded floating beads in chronic fatigue syndrome: behavioral and biochemical evidences. Neurogastroenterol Motil 2012;24:366-e170.

41. Arseneault-Bréard J, Rondeau I, Gilbert K, et al. Combination of Lactobacillus helveticus R0052 and Bifidobacterium longum R0175 reduces post-myocardial infarction depression symptoms and restores intestinal permeability in a rat model. Br J Nutr 2012;107:1793-1799.

42. Luo J, Wang T, Liang S, Hu X, Li W, Jin F. Ingestion of Lactobacillus strain reduces anxiety and improves cognitive function in the hyperammonemia rat. Sci China Life Sci 2014;57:327-335.

43. Savignac HM, Tramullas M, Kiely B, Dinan TG, Cryan JF. Bifidobacteria modulate cognitive processes in an anxious mouse strain. Behav Brain Res 2015;287:59-72.

44. Liu J, Sun J, Wang F, et al. Neuroprotective Effects of Clostridium butyricum against Vascular Dementia in Mice via Metabolic Butyrate. Biomed Res Int 2015;2015:412946.

45. Gareau MG, Wine E, Rodrigues DM, et al. Bacterial infection causes stress-induced memory dysfunction in mice. Gut 2011;60:307-317.

46. Davari S, Talaei SA, Alaei H, Salami M. Probiotics treatment improves diabetes-induced impairment of synaptic activity and cognitive function: behavioral and electrophysiological proofs for microbiome-gut-brain axis. Neuroscience 2013;240:287-296.

47. Jeong JJ, Kim KA, Ahn YT, et al. Probiotic mixture KF attenuates agedependent memory deficit and lipidemia in Fischer 344 rats. J Microbiol Biotechnol 2015;25:1532-1536.

48. Kantak PA, Bobrow DN, Nyby JG. Obsessive-compulsive-like behaviors in house mice are attenuated by a probiotic (Lactobacillus rhamnosus GG). Behav Pharmacol 2014;25:71-79.

49. D’Mello C, Ronaghan N, Zaheer R, et al. Probiotics improve inflammation-associated sickness behavior by altering communication between the peripheral immune system and the brain. J Neurosci 2015;35:1082110830.

50. Takada M, Nishida K, Kataoka-Kato A, et al. Probiotic Lactobacillus casei strain Shirota relieves stress-associated symptoms by modulating the gut-brain interaction in human and animal models. Neurogastroenterol Motil 2016;28:1027-1036.

51. Rao AV, Bested AC, Beaulne TM, et al. A randomized, double-blind, placebo-controlled pilot study of a probiotic in emotional symptoms of chronic fatigue syndrome. Gut Pathog 2009;1:6.

52. Mohammadi AA, Jazayeri S, Khosravi-Darani K, et al. The effects of probiotics on mental health and hypothalamic-pituitary-adrenal axis: a randomized, double-blind, placebo-controlled trial in petrochemical workers. Nutr Neurosci Published Online First: 16 Apr 2015. doi: 10.1179/1476830515Y.0000000023.

53. Steenbergen L, Sellaro R, van Hemert S, Bosch JA, Colzato LS. A randomized controlled trial to test the effect of multispecies probiotics on cognitive reactivity to sad mood. Brain Behav Immun 2015;48:258-264.

54. Dickerson FB, Stallings C, Origoni A, et al. Effect of probiotic supplementation on schizophrenia symptoms and association with gastrointestinal functioning: a randomized, placebo-controlled trial. Prim Care Companion CNS Disord Published Online First: 13 Feb 2014. doi: 10.4088/ PCC. $13 \mathrm{~m} 01579$.

55. Vaghef-Mehrabany E, Alipour B, Homayouni-Rad A, Sharif SK, Asghari-Jafarabadi M, Zavvari S. Probiotic supplementation improves inflammatory status in patients with rheumatoid arthritis. Nutrition 2014;30:430-435.

56. Dapoigny M, Piche T, Ducrotte P, Lunaud B, Cardot JM, BernalierDonadille A. Efficacy and safety profile of LCR35 complete freeze-dried culture in irritable bowel syndrome: a randomized, double-blind study. World J Gastroenterol 2012;18:2067-2075.

57. Simrén M, Ohman L, Olsson J, et al. Clinical trial: the effects of a fermented milk containing three probiotic bacteria in patients with irritable bowel syndrome - a randomized, double-blind, controlled study. Aliment Pharmacol Ther 2010;31:218-227.

58. Whorwell PJ, Altringer L, Morel J, et al. Efficacy of an encapsulated probiotic Bifidobacterium infantis 35624 in women with irritable bowel syndrome. Am J Gastroenterol 2006;101:1581-1590.

59. Reale M, Boscolo P, Bellante V, et al. Daily intake of Lactobacillus casei Shirota increases natural killer cell activity in smokers. Br J Nutr 2012;108:308-314.

60. Parracho HMRT, Gibson GR, Knott F, Bosscher D, Kleerebezem M, McCartney AL. A double blind, placebo controlled, crossover designed 
probiotic feeding study in children diagnosed with autistic spectrum disorders. International Journal of Probiotics and Prebiotics 2010;5:69.

61. Messaoudi M, Violle N, Bisson JF, Desor D, Javelot H, Rougeot C. Beneficial psychological effects of a probiotic formulation (Lactobacillus helveticus R0052 and Bifidobacterium longum R0175) in healthy human volunteers. Gut Microbes 2011;2:256-261.

62. Benton D, Williams C, Brown A. Impact of consuming a milk drink containing a probiotic on mood and cognition. Eur J Clin Nutr 2007;61:355-361.

63. Tillisch K, Labus J, Kilpatrick L, et al. Consumption of fermented milk product with probiotic modulates brain activity. Gastroenterology 2013;144:1394-1401, e1-e4.

64. Sung V, Collett S, de Gooyer T, Hiscock H, Tang M, Wake M. Probiotics to prevent or treat excessive infant crying: systematic review and metaanalysis. JAMA Pediatr 2013;167:1150-1157.

65. Romijn AR, Rucklidge JJ. Systematic review of evidence to support the theory of psychobiotics. Nutr Rev 2015;73:675-693.

66. Anabrees J, Indrio F, Paes B, AlFaleh K. Probiotics for infantile colic: a systematic review. BMC Pediatr 2013;13:186.

67. Groeger D, O'Mahony L, Murphy EF, et al. Bifidobacterium infantis 35624 modulates host inflammatory processes beyond the gut. Gut Microbes 2013;4:325-339.

68. Lomasney KW, Cryan JF, Hyland NP. Converging effects of a Bifidobacterium and Lactobacillus probiotic strain on mouse intestinal physiology. Am J Physiol Gastrointest Liver Physiol 2014;307:G241-G247.

69. Saulnier DM, Ringel Y, Heyman MB, et al. The intestinal microbiome, probiotics and prebiotics in neurogastroenterology. Gut Microbes 2013;4:17-27.

70. O'Mahony L, McCarthy J, Kelly P, et al. Lactobacillus and Bifidobacterium in irritable bowel syndrome: symptom responses and relationship to cytokine profiles. Gastroenterology 2005;128:541-551.

71. McFarland LV. From yaks to yogurt: the history, development, and current use of probiotics. Clin Infect Dis 2015;60(suppl 2):S85-S90.

72. Floch MH, Walker WA, Sanders ME, et al. Recommendations for probiotic use--2015 update: proceedings and consensus opinion. J Clin Gastroenterol 2015;49(suppl 1):S69-S73.

73. Dedovic K, Ngiam J. The cortisol awakening response and major depression: examining the evidence. Neuropsychiatr Dis Treat 2015;11:11811189.

74. Osborne DM, Pearson-Leary J, McNay EC. The neuroenergetics of stress hormones in the hippocampus and implications for memory. Front Neurosci 2015;9:164.

75. Mariga A, Mitre M, Chao MV. Consequences of brain-derived neurotrophic factor withdrawal in CNS neurons and implications in disease. Neurobiol Dis Published Online First: 22 Mar 2016. doi: 10.1016/ j.nbd.2016.03.009.

76. Tsai SJ. Attention-deficit hyperactivity disorder may be associated with decreased central brain-derived neurotrophic factor activity: clinical and therapeutic implications. Med Hypotheses 2007;68:896-899.

77. Julio-Pieper M, Bravo JA, Aliaga E, Gotteland M. Review article: intestinal barrier dysfunction and central nervous system disorders - a controversial association. Aliment Pharmacol Ther 2014;40:1187-1201.
78. Desbonnet L, Clarke G, Shanahan F, Dinan TG, Cryan JF. Microbiota is essential for social development in the mouse. Mol Psychiatry 2014;19:146-148.

79. Diaz Heijtz R, Wang S, Anuar F, et al. Normal gut microbiota modulates brain development and behavior. Proc Natl Acad Sci USA 2011;108:3047-3052.

80. Lyte M, Li W, Opitz N, Gaykema RP, Goehler LE. Induction of anxiety-like behavior in mice during the initial stages of infection with the agent of murine colonic hyperplasia Citrobacter rodentium. Physiol Behav 2006;89:350-357.

81. Neufeld KM, Kang N, Bienenstock J, Foster JA. Reduced anxiety-like behavior and central neurochemical change in germ-free mice. Neurogastroenterol Motil 2011;23:255-264, e119.

82. Nishino R, Mikami K, Takahashi H, et al. Commensal microbiota modulate murine behaviors in a strictly contamination-free environment confirmed by culture-based methods. Neurogastroenterol Motil 2013;25:521-528.

83. Astur RS, Ortiz ML, Sutherland RJ. A characterization of performance by men and women in a virtual Morris water task: a large and reliable sex difference. Behav Brain Res 1998;93:185-190.

84. Astur RS, Taylor LB, Mamelak AN, Philpott L, Sutherland RJ. Humans with hippocampus damage display severe spatial memory impairments in a virtual Morris water task. Behav Brain Res 2002;132:77-84.

85. Kalová E, Vlcek K, Jarolímová E, Bures J. Allothetic orientation and sequential ordering of places is impaired in early stages of Alzheimer's disease: corresponding results in real space tests and computer tests. Behav Brain Res 2005;159:175-186.

86. Laczó J, Andel R, Vyhnalek M, et al. Human analogue of the morris water maze for testing subjects at risk of Alzheimer's disease. Neurodegener Dis 2010;7:148-152.

87. Kirschbaum C, Pirke KM, Hellhammer DH. The 'Trier Social Stress Test' - a tool for investigating psychobiological stress responses in a laboratory setting. Neuropsychobiology 1993;28:76-81.

88. Sebastian C, Viding E, Williams KD, Blakemore SJ. Social brain development and the affective consequences of ostracism in adolescence. Brain Cogn 2010;72:134-145.

89. Williams KD, Jarvis B. Cyberball: a program for use in research on interpersonal ostracism and acceptance. Behav Res Methods 2006;38:174180.

90. Allen AP, Kennedy PJ, Cryan JF, Dinan TG, Clarke G. Biological and psychological markers of stress in humans: focus on the Trier Social Stress Test. Neurosci Biobehav Rev 2014;38:94-124.

91. Schwabe L, Haddad L, Schachinger H. HPA axis activation by a socially evaluated cold-pressor test. Psychoneuroendocrinology 2008;33:890895.

92. Cian C, Barraud PA, Melin B, Raphel C. Effects of fluid ingestion on cognitive function after heat stress or exercise-induced dehydration. Int $\mathrm{J}$ Psychophysiol 2001;42:243-251.

93. Vickers K, Jafarpour S, Mofidi A, Rafat B, Woznica A. The 35\% carbon dioxide test in stress and panic research: overview of effects and integration of findings. Clin Psychol Rev 2012;32:153-164. 